\title{
Macrophage migration inhibitory factor is a novel structure component of centrioles and a novel transcriptional factor
}

\section{Lu Zhang}

Mayo Clinic

Hongbing Zhang

Mayo Clinic

Xiaoyan Li

Mayo Clinic https://orcid.org/0000-0002-7460-0776

Ewud Agborbesong

Mayo Clinic

Xiaogang Li ( $\sim$ li.xiaogang@mayo.edu )

Mayo Clinic https://orcid.org/0000-0001-8135-342X

\section{Article}

Keywords: ciliopathies, cilia, macrophage migration inhibitory factor (MIF)

Posted Date: December 29th, 2020

DOI: https://doi.org/10.21203/rs.3.rs-126373/v1

License: (c) (1) This work is licensed under a Creative Commons Attribution 4.0 International License. Read Full License 


\section{Abstract}

Ciliopathies are a group of human diseases that affect the cellular cilia or the cilia anchoring structures, the bosal bodies, or ciliary function. Macrophage migration inhibitory factor (MIF) as an important inflammatory cytokine plays a prominent role in the pathogenesis of various human diseases. However, the role of MIF in ciliopathies remains elusive. In this study, we show that MIF is a structure protein of basal bodies, which forms a ring-like structure at proximal end of centrioles to regulate cilia assembly and elongation. Importantly, we identify MIF as a novel transcriptional factor, which regulates the expression of ciliary genes and genes associated with different signaling pathways. The phosphatidylinositol-5-phosphate 4-kinase type 2 alpha (PIP4K2a) mediated MIF phosphorylation at S91 is necessary for the nuclear translocation of MIF, a process that is regulated by $14-3-3 \zeta$. This study suggests that MIF is a key player in cilia biogenesis and transcriptional regulation in homeostasis.

\section{Introduction}

Macrophage migration inhibitory factor (MIF), which is encoded by the MIF gene, has been classified as a preinflammatory cytokine and is involved in cell-mediated immunity, immunoregulation, and inflammation [1]. As a unique cytokine with respect to its versatile features, MIF is constitutively and ubiquitously expressed in both immune and non-immune cells, such as monocytes/macrophages, B- and T-cells, and endothelial and epithelial cells, in response to stress caused by different factors, leading to pathological conditions [1, 2]. MIF can be released from blood cells into the blood stream. The circulating MIF binds to the extracellular domain of CD74, a cell surface receptor for MIF [3], on other immune cells to trigger an acute immune response. MIF could also function as a noncognate ligand for the chemokine receptors CXCR2 and CXCR4 which may be coordinated by CD74, and thereby controls inflammation and leukocyte recruitment [2-4].

Besides regulation of immunity and inflammation, MIF has also been implicated in cell proliferation, cell cycle progress, apoptosis, and even tumor growth, metastasis and angiogenesis [3,5-8]. Upregulation of MIF results in imbalance of homeostasis in almost all types of human cancers and is implicated in seemingly all stages of development of the tumors. Genetic studies demonstrated that MIF promotes Bcell lymphomagenesis and intestinal tumorigenesis in mice $[5,9]$. Conversely, depletion of MIF in animal models impaired tumor growth $[10,11]$. The production of MIF is triggered through an autocrine signal emitted by tumor cells, and stimulates the production of cytokines and chemokines that lead to growth of the tumor, increasing its aggressiveness and metastatic potential. In addition, MIF has a wide spectrum of biological activities and interacts with various cellular signaling pathways, including ERK/MAPK, PI3K/Akt, NF-KB and c-Myc-dependent pathway as well as TGF- $\beta$ and p53-dependent pathway $[6,7,9,12]$. Targeting MIF decreased Rb-E2F, NF-KB, c-Myc and Ap-1-mediated transcription and increased p53 and FoxO4 transcriptional activity, leading to differential expression of cell cycle regulators and subsequent cell cycle arrest in G0/G1 phase [12]. However, whether MIF by itself functions as a transcriptional factor has not yet been elucidated. 
The biogenesis and resorption of primary cilia are intimately associated with the cell cycle, as primary cilia form during the G0/G1 phase (quiescence) and resorb before mitosis [13]. Primary cilium is a nonmotile sensory and signaling organelle, which is present in diverse cell types in human body [14]. Primary cilium is initiated in a specific region of the plasma membrane, the entire cilia structure is enclosed by the cell plasma membrane, while inside cilia is a microtubule-based cytoskeleton structure called the axoneme which is grown from the basal body $[15,16]$. Primary cilia function as cellular antennae on eukaryotic cells to sense specific signaling cues from the extracellular environment, including mechanosensation, chemosensation and thermosensation [17]. Genetic diseases where the assembly and function of primary cilia are defective, ciliopathies, indicate that primary cilia are tightly associated with normal development in mammals [18]. The role of MIF in G1 phase to drive quiescent cells into $S$ phase and cilia formation in the G0/G1 phase implies an intrinsic connection of MIF with ciliogenesis during the first gap phase of the cell cycle.

In this study, we investigated the roles and mechanisms of MIF in cilia biogenesis and demonstrated a novel role of MIF in transcriptional regulation of genes related to ciliogenesis and other signaling pathways. We found that MIF is localized and formed a ring like structure at the proximal end of centrioles. MIF regulates cilia biogenesis through affecting 1) the recruitment of TTBK2 to basal body and the removal of CP110 from mother centriole, 2) the accumulation of CEP290 and BBS4 at centriolar satellites, 3) the translocation of HYLS-1 from basal body into axoneme via their interaction, 4) the trafficking of intraflagellar transport (IFT) related proteins, and 5) the transcription of genes associated with ciliogenesis. Importantly, MIF also binds chromatin and regulates transcription of genes involved in diverse homeostatic signaling pathways. Further analysis of those MIF target genes should facilitate our understanding of the roles and mechanisms of MIF in tumorigenesis and ciliopathies.

\section{Results}

\section{The expression of MIF is dynamically associated with cilia assembly and disassembly during cell cycle.}

Cilia assembly and disassembly is a dynamic process during cell cycle, in that cilium is initiated to be assembled at G0/G1 phase, and usually present in S/G2 phase, but almost invariably resorbed before mitotic entry, and to re-appear post-cytokinesis [19]. To investigate whether the expression of MIF changes during cell cycle, we treated human renal cortical tubular epithelial (RCTE) cells with thymidinenocodazole or double thymidine block which could synchronize cells at G2/M or G1/S phase (Fig. 1a), respectively. After removal of these inhibitors, we collected cells at the different time points (Fig. 1b). The synchronization of cells with indicated inhibitors was confirmed by FACS analysis (Fig. 1c, d). We found that the expression of MIF, Cyclin B, Cdc20 and $\mathrm{CDH} 1$ were fluctuated in thymidine-nocodazole synchronized RCTE cells during cell cycle, which was peaked in G2/M phases, and then sharply reduced in $\mathrm{G} 1$ phase (Fig. 1e). When RCTE cells were synchronized with double thymidine block at G1/S phase, the expression of MIF as well as Cyclin B, Cdc20 and $\mathrm{CDH} 1$ was also peaked in $\mathrm{G} 2 / \mathrm{M}$ phase and reduced in $\mathrm{G} 1$ phase during cell cycle (Fig. 1f). In addition, we found that the expression of MIF, Cyclin B, Cdc20 and $\mathrm{CDH} 1$ was also peaked in G2/M phases and reduced in $\mathrm{G} 1$ phase during cell cycle in Hela cells 
synchronized with either thymidine-nocodazole or double thymidine block (Supplementary Fig. 1a-d). The reduction of MIF at G1 phase and the increase of MIF at G2/M phase are associated with cilia assembly and disassembly during cell cycle, suggesting that MIF may play a role in these processes.

\section{MIF forms a ring-like structure at proximal end of the centrioles.}

To investigate whether MIF plays a role in cilia biogenesis, first, we determined the subcellular localization of MIF in RCTE and human pigment epithelial (RPE) cells. We found that MIF was localized at basal bodies (centrioles) marked with $y$-tubulin and pericentrin but not along axoneme marked with a-acetyltubulin in RCTE (Fig. 1g-j) and RPE cells (Supplementary Fig. 1e-h). The localization of MIF at basal bodies was verified with a second MIF antibody in RCTE and RPE cells co-stained with $\mathrm{y}$-tubulin and Arl13b, another axoneme marker (Supplementary Fig. 1i, j). With 3D structured illumination microscopy (3D-SIM), we found that MIF formed a ring-like structure around the centrioles (both mother and daughter centrioles) in RCTE cells (Fig. 1g, j) and RPE cells (Supplementary Fig. 1e, h). To determine a more precise localization of MIF at centrioles, we co-stained MIF with different centriolar/centrosomal markers, including CEP164 (a marker for a distal appendage [20]), Ninein (a marker for sub-distal appendages of mother centrioles and proximal ends of both centrioles [21]) and y-tubulin (a marker for pericentriolar materials) in RCTE cells. Under 2D-microscopy, MIF co-localized with all these markers, in that MIF was co-localized with y-tubulin (Fig. 1g) and Ninein (Fig. 2a), but was localized adjacent to CEP164 (Fig. 2b). Under 3D-SIM, we found that MIF was not colocalized with CEP164 (Fig. 2c-e) in RCTE cells, suggesting that MIF was not localized at or around the distal appendages, whereas MIF was colocalized with Ninein around proximal end of mother and daughter centrioles (Fig. 2f-h) in those cells. To further support the localization of MIF at the proximal end of centrioles, we co-stained MIF with C-Nap1 (centrosomal NEK2associated protein 1) which anchors rootletin and CEP68 filaments, respectively, to the proximal ends of the two centrioles and is involved in the regulation of procentriole formation during late G1/S phase [21]. We found a ring-like structure of MIF surrounding the proximal end of centriole marked with C-Nap1 (Fig. 2i-k). These results suggested that MIF is a proximal end protein of both centrioles as indicated in the diagram (Fig. 2l).

In mammalian cells, centrioles are $\sim 230 \mathrm{~nm}$ in diameter and $\sim 500 \mathrm{~nm}$ in length [22]. We determined the diameters of ring structures of MIF, CEP164, Ninein and $\gamma$-tubulin by measuring the distances between intensity maximal (Supplementary Fig. 2a-d, right panel). We found that the diameter of MIF rings was about $300 \mathrm{~nm}$, which was smaller than the diameters of $\mathrm{Y}$-tubulin (401.3 \pm 14.2$)$ and CEP164 (409.1 \pm $9.4 \mathrm{~nm}$ ) rings, and was much smaller than the diameter of Ninein ring (590 $\pm 13.2 \mathrm{~nm}$ ) (Supplementary Fig. 2e).

In this study, we localized PACRG by immuno-electron microscopy for the first time, and show that its epitope is frequently found between adjacent outer doublets (Fig. 3B), an observation that supports the involvement of PACRG in the inter-doublet linkage In this study, we localized PACRG by immuno-electron microscopy for the first time, and show that its epitope is frequently found between adjacent outer doublets (Fig. 3B), an observation that supports the involvement of PACRG in the inter-doublet 
linkage we localized PACRG by immuno-electron microscopy for the first time, and show that its epitope is frequently found between adjacent outer doublets

\section{MIF is a critical regulator of cilia formation and elongation.}

To support a role of MIF on ciliogenesis, we found that 1) knockdown of MIF (Fig. 3a-d) increased the number of ciliated cells and cilia length in RCTE (Fig. 3e, f) and RPE (Fig. 3g, h) cells compared to those in the control siRNA transfected cells, 2) treatment with MIF inhibitor, ISO-1, also increased the numbers of ciliated cells and cilia length in RCTE (Fig. $3 \mathrm{i}, \mathrm{j}$ ) and RPE (Fig. 3k, I) cells compared to those in control cells treated with DMSO, and 3) treatment with purified human recombinant MIF (rhMIF) decreased the numbers of ciliated cells and cilia length in RCTE cells compared to that in control cells treated with PBS (Supplementary Fig. 3a, b).

To elucidate the role of MIF in ciliogenesis in vivo, we examined ciliogenesis in cilia-rich mouse kidneys. We found that knockout of Mif (Supplementary Fig. 3c) strikingly increased cilia length and ciliated cells in lumens of proximal and distal tubules in kidneys collected from 21 days (Supplementary Fig. 4a, b) and 3 months old Mif knockout mice (Supplementary Fig. 4c, d) compared to those in the lumens of tubules in kidneys from age matched Mif wild type mice. We further found that knockout of Mif increased cilia length and ciliated cells in primary renal epithelial cells isolated from Mif knockout kidneys compared to that in Mif wild type renal epithelial cells isolated from age matched wild type kidneys (Supplementary Fig. 4e-h). These results suggest that MIF functions as a critical regulator of cilia biogenesis in vitro and in vivo.

\section{MIF blocks cilia elongation via inhibiting CP110 removal and TTBK2 recruitment.}

Cilia assembly and elongation require the removal of the CP110, a centrosomal protein, from the distal end of mother centriole to allow the growth of axonemal microtubules [23]. We found that depletion of MIF resulted in the removal of CP110 from mother centriole in RCTE cells (Supplementary Fig. 5a, c) and RPE cells (Supplementary Fig. 5b, d) compared to its presence on both centrioles in the control cells. We further found that depletion of MIF did not affect the localization of $y$-tubulin, which was detected on both centrioles as marked as two dots in RCTE cells (Supplementary Fig. 5e, g) and RPE cells (Supplementary Fig. 5f, h), suggesting that MIF depletion did not disturb centrosomes in those cells. The kinase TTBK2 mediates the removal and dissociation of CP110 from the CP110 and CEP290 complex at the mother centriole, leading to the formation of the primary cilium [24]. We found that knockdown of MIF increased the recruitment of TTBK2 to basal body in RCTE cells (Supplementary Fig. 5i, j). These results suggest that MIF may control cilia assembly and elongation by increasing the recruitment of CP110 to the mother centriole and decreasing the recruitment of TTBK2 to basal bodies.

\section{Knockdown of MIF increases the accumulation of CEP290 and BBS4 at centriolar satellites.}

It has been reported that the interaction of CP110 with CEP290 antagonizes the action of CEP290, which in turn prevents ciliogenesis [25]. CEP290 is localized on both mother and daughter centrioles, where it is 
maintained in an inhibited state by CP110 [25]. We found that knockdown of MIF resulted in an increase of centriolar satellite-associated CEP290 around basal body in RCTE cells (Supplementary Fig. 6a, b). It has been reported that the centriolar satellites located Cep290 may facilitated the re-localization of BBS4 from the satellites to the cilium [26]. We found that depletion of MIF resulted in an increase of centriolar satellite-associated BBS4 and the entry of BBS4 to axoneme (Supplementary Fig. 6c, d). These results suggest that depletion of MIF increases the accumulation of CEP290 and BBS4 in centriolar satellites, which then regulates the ciliary localization of BBS4 to possibly affect cilia assembly.

\section{MIF interacts with HYLS-1 to prevent HYLS-1 translocation from basal body into axoneme.}

HYLS-1 as a conserved centrioles and basal body component is required for the docking and entry of intraflagellar transport (IFT) particles in C. elegans [27]. We found that HYSL-1 also formed a ring like structure and was co-localized with CEP135 (another marker for the proximal region of the centriole [28]) (Fig. 4a-c) and pericentrin (another maker for pericentriolar material) (Fig. 4d-f) in RCTE cells, whereas HYLS-1 was distant to CP110 that was located at distal end of centrioles (Fig. 4g-i), suggesting that HYLS-1 is a centriolar proximal end protein in mammalian cells. We further found that MIF interacted with HYLS-1 in RCTE cells as examined by co-immunoprecipitation analysis (Fig. 4j). We also found that MIF was co-localized with HYLS-1 at the proximal end of both centrioles in serum starved RCTE cells under 2D and 3D microscopy (Fig. 4k-m), which further supported that MIF is located at proximal end of centrioles (Fig. 4n). To understand the role of MIF and HYLS-1 interaction in cilia biogenesis, we found that knockdown of MIF (Fig. 4o, p) and inhibition of MIF with ISO-1 (Fig. 4q, r) resulted in the translocation of HYLS-1 from basal body into the axoneme in RCTE cells. These results indicated for the first time that HYLS-1 could enter the axoneme in mammalian cells, which may promote cilia assembly and elongation, and suggested that MIF might function upstream of HYLS-1 to block its entry to axoneme and then affect cilia elongation.

\section{Loss of MIF promotes IFT related proteins recruitment and entry.}

For that MIF is involved in regulation of ciliary localization of TTBK2, BBS4 and HYLS-1, and all of these proteins regulate the trafficking of cilia associated proteins $[27,29,30]$, we examined whether targeting MIF affects the trafficking of IFTs and their associated motor proteins in RCTE cells. We found that knockdown of MIF and inhibition of MIF with ISO-1 resulted in a cilia tip accumulation of KIF3a, an essential subunit of the kinesin II motor that is required for cilia formation in nearly all cells [31], in RCTE cells, which was in contrast to its basal body localization in control cells (Supplementary Fig. 7a-c and Supplementary Fig. 8a-c). The accumulation of KIF3a at ciliary tips suggested an increase of anterograde trafficking of cilia proteins, which is regulated by IFT B complex, and a decrease of retrograde trafficking of these proteins, which is regulated by IFT A complex, in MIF knockdown cells. To support this, we found that knockdown of MIF and inhibition of MIF with ISO-1 resulted in an increase of the accumulation of IFT20 at the cilia tips in RCTE cells (Supplementary Fig. 7d-f and Supplementary Fig. 8d-f). We also found that knockdown of MIF and inhibition of MIF with ISO-1 increased the intensity of IFT88 at ciliary tips, and induced a punctate distribution of IFT88 along cilia in RCTE cells (Supplementary Fig. 7g-I and 
Supplementary Fig. 8g-i). Both IFT20 and IFT88 belong to IFT B complex. In addition, we found that knockdown of MIF and inhibition of MIF with ISO-1 decreased the tip location of a core IFT A protein, IFT140, in RCTE cells (Supplementary Fig. 7j-I and Supplementary Fig. 8j-I). Unexpectedly but importantly, we found that knockdown of MIF (Supplementary Fig. 9a-c) and inhibition of MIF with ISO-1

(Supplementary Fig. 9d-f) increased the mRNA and protein levels of KIF3a and IFT20 in RCTE cells. These results suggest that MIF regulates the trafficking of KIF3a and IFT proteins, and may also negatively regulate the transcription of KIF3a and IFT20 and other genes related to ciliary biogenesis.

\section{MIF regulates ciliary gene transcription through binding on the promoters of those genes.}

To support a role of MIF in transcription regulation, we found that knockdown of MIF also increased the transcription of genes of IFT A particles (IFT121, IFT139, IFT140, IFT144), IFT B particles (IFT27, IFT88), centriolar satellite/ transition zone protein (CEP290), transition zone protein (TTBK2), distal appendage protein (CEP89), basal body and axoneme protein (Rab8a), basal body protein (OFD1) and BBSome protein (BBS4) (Supplementary Fig. 10a), whereas treatment with rhMIF decreased the transcription of those genes (Supplementary Fig. 10b). We further found that MIF could bind on the promoters of the tested genes, including KIF3a, IFT20, CEP290 and BBS4 (Supplementary Fig. 11a-d). These results suggest that MIF may be a novel transcriptional factor to negatively regulate the transcription of ciliary genes.

\section{Identification of MIF target genes with ChIP-sequencing (ChIP-seq) analysis.}

We identified the genome wide MIF target genes with ChIP-seq analysis. We found that there were about $14 \%$ of reproducible and statistically significant MIF binding peaks located within gene promoter regions (0-1000 bp) (Fig. 5a), which were markedly concentrated near the TSS sites (Fig. 5b). We summarized the potential MIF target genes related to ciliogenesis in Supplementary Data 1. As an example, we showed the identified peaks in ChIP-seq analysis corresponding to the binding of MIF on the promoters of KIF3a, IFT20, CEP290 and BBS4 genes (Fig. 5c-f, black boxes), which was confirmed with ChIP assay above (Supplementary Fig. 11a-d). These results further supported that MIF is a novel transcriptional factor, which may regulate cilia biogenesis via regulating the transcription of ciliary genes.

\section{MIF also regulates the transcription of genes in diverse signaling pathways.}

The MIF ChIP-seq analysis also identified genes other than those related to cilia biogenesis (Supplementary Data 2). With Kyoto Encyclopedia of Genes and Genomes (KEGG) pathway-based analysis, we found that the identified MIF target genes associated signaling pathways of spliceosome, RNA transport, cell cycle, ribosome, autophagy, protein processing in endoplasmic reticulum, Parkinson disease, mitophagy, ubiquitin mediated proteolysis, etc. (Fig. 5g). With GREAT (Genomic Regions Enrichment of Annotations Tool) analysis, a tool that allows us to find enriched ontological terms in a set of genomic regions to better understand the underlying biological processes, we also clustered MIF occupied genes with a range of gene ontology (GO) biological processes, including autophagy, cell cycle transition signaling, and cell cycle checkpoint pathways, etc. (Supplementary Fig. 12). The GO cellular 
component analysis also revealed that MIF target genes were highly enriched for cilia-associated components, such as centrosome, and other cellular structures, such as nuclear envelope, nuclear membrane, nuclear speck and nuclear chromatin, etc. (Fig. 5h). These analyses suggested that MIF regulated the expression of genes associated with cilia biogenesis and homeostasis through directly binding on the promoters of those genes.

\section{The translocation of MIF to nucleus is dependent on its interaction with 14-3-3.}

In order to act as a transcriptional factor, MIF has to be translocated from cytosol to nucleus. To identify a MIF binding partner that facilitates its translocation into nucleus, we performed protein chip by using a human recombinant MIF protein to screen its interacting proteins on HuProtTM human protein chip which contains more than 21,000 human recombinant proteins (See Methods) (Fig. 6a). In brief, rhMIF or blocking buffer was probed on the human protein chip, and then the microarray was further incubated with a Cy3-conjugated antibody to present the MIF-protein interactions. With this analysis, we identified 225 proteins as potential binding partners of MIF (Supplementary Data 3 ).

In addition, we identified the proteins that interacted with MIF by performing immunoprecipitation with MIF antibody and followed by mass spectrometry (MS) analysis in RCTE cells (Fig. 6b). With this analysis we identified 512 proteins as potential binding partners of MIF (Supplementary Data 4), including YWHAZ (14-3-3ろ), PIP4K2a, etc. Functional annotation analysis revealed that the MIF interaction proteins were significantly enriched for proteins involved in protein ubiquitination $(p<0.0032)$, protein phosphorylation $(p<0.017)$, apoptotic process $(p<0.0459)$, and MAPK cascade $(p<0.0016)$, many of which affect protein activity (Fig. 6c).

14-3-3 is highly conserved and can be self-assemble into homo- and heterodimers with a diverse array of cellular proteins, including transcription factors, biosynthetic enzymes, cytoskeletal proteins, apoptosis factors and tumor suppressors [32]. We confirmed that MIF interacted with 14-3-3 3 in RCTE cells by co-IP assay (Fig. 6d). We found that MIF could be detected in nuclear and cytosol fractions in RCTE cells (Fig. 6e), whereas knockdown of 14-3-3ろ with siRNA decreased the levels of MIF in nuclear fraction but not that in cytosol fraction (Fig. 6f), suggesting that the binding of MIF with 14-3-3 3 might promote it nuclear transportation.

With the alignment of MIF amino acid sequence with a conserved 14-3-3 binding motif, RXXXS [33], we identified that RXXXS91 of MIF is a potential 14-3-3 binding site (Fig. $6 \mathrm{~g}$ ). It has been reported that the phosphorylation of serine in RXXXS motif facilitates the nuclear translocation of 14-3-3 partner(s). To test this possibility, we generated MIF mutant constructs by replacing the serine 91 of MIF with aspartic acid (D) (constantly phosphorylated mutation) or alanine (A) (un-phosphorylated mutation) (Fig. 6h). We found that the interaction between GFP tagged mutant MIF (S91D) and 14-3-3ろ was increased compared to that between GFP tagged wild type MIF and 14-3-3 3 in RCTE cells (Fig. 6i). However, we found a negligible interaction between GFP tagged mutant MIF (S91A) and 14-3-3Z compared to the interaction between GFP tagged wild type MIF and 14-3-3ろ (Fig. 6j). Treatment with lambda phosphatase also 
decreased the interaction of GFP-tagged wild type MIF with 14-3-3ろ (Fig. 6k). These results suggested that the interaction between MIF and 14-3-3ろ might be dependent on the phosphorylation of MIF at S91.

\section{Phosphorylation of MIF at Ser91 affects its nuclear translocation and its role in ciliogenesis.}

To investigate whether phosphorylation of MIF at S91 is involved in its nuclear translocation/exportation, we transfected GFP-tagged wild type MIF and mutant MIF (S91D or S91A) into HEK293T cells. We found that GFP tagged wild-type MIF and mutant MIF at S91A was mostly located in cytosol, whereas the GFP tagged MIF S91D mutant was located in both nucleus and cytosol in GFP positive cells as examined with immunostaining analysis (Fig. 6l, m). We further found that the level of the GFP tagged mutant MIF at S91D was increased in nuclear fraction compared to that in nuclear fraction of cells transfected with GFP tagged wild-type MIF (Fig. 6n, o), whereas the nuclear transportation of mutant MIF S91A was blocked (Fig. 6l, n). In addition, we found that the expression of cilia associated genes, including KIF3A, IFT20, CEP290 and BBS4, and the percentage of ciliated cells and cilia length were significantly decreased in RCTE (Supplementary Fig. 13a-g) and RPE (Supplementary Fig. 14a-g) cells transfected with GFP tagged wild type MIF and MIFS91D compared to those in cells transfected with GFP-vector and GFP tagged MIFS91A. These results support that the phosphorylation of MIF at Ser91 is required for MIF nuclear translocation and MIF mediated cilia biogenesis.

\section{PIP4K2a interacts with MIF and induces the phosphorylation of MIF at Ser91.}

Our protein chip and mass spectrometry (MS) analysis identified that PIP4K2a is a potential kinase responsible for the phosphorylation of MIF. To support this hypothesis, we confirmed that PIP4K2a interacted with MIF and 14-3-3 $\zeta$ in RCTE cells as examined with co-IP assay (Fig. 7a, b). We further found that knockdown of PIP4K2a decreased the phosphorylation of MIF, and decreased the interaction of MIF with 14-3-3Z in RCTE cells (Fig. 7c), whereas overexpression of GFP tagged PIP4K2a increased the phosphorylation of MIF and the interaction of MIF with 14-3-3Z in RCTE cells (Fig. 7d), suggesting a crosstalk between PIP4K2a and MIF as well as phospho-MIF and 14-3-3 3 in MIF nuclear translocation (Fig. 7e). Knockdown and overexpression of PIP4K2a has no effect on the expression of MIF in RCTE (Supplementary Fig. 15a, b) and RPE cells (Supplementary Fig. 16a, b). However, knockdown PIP4K2a increased the transcription of ciliary genes, including KIF3a, IFT20, IFT88, TTBK2, CEP290 and BBS4 in RCTE (Supplementary Fig. 15a) and RPE cells (Supplementary Fig. 16a), and overexpression of PIP4K2a decreased the transcription of those ciliary genes in RCTE (Supplementary Fig. 15b) and RPE cells (Supplementary Fig. 16b). Importantly, we found that knockdown of PIP4K2a with siRNA also increased cilia length and ciliated cell population in RCTE cells (Fig. 7f, g) and RPE cells (Fig. 7h, i), whereas overexpression of GFP tagged PIP4K2A decreased cilia length and ciliated cell population in RCTE cells (Fig. 7j, k) and RPE cells (Fig. 7l, m). These results suggest that PIP4K2a is an upstream regulator of MIF, which may increase its interaction with 14-3-3ろ, resulting in its nuclear translocation to execute it transcriptional function to regulate cilia biogenesis.

\section{Discussion}


MIF as an inflammatory cytokine is expressed broadly in different types of human cells. In addition to its roles in diverse inflammatory processes, MIF also regulates tumorigenesis and cystogenesis via binding on its receptors to affect diverse signaling pathways in cancer cells and cystic cells [6,7]. Thus, MIF has emerged as a promising drug target in diseases including sepsis, rheumatoid arthritis, polycystic kidney disease (PKD) and cancer. However, whether MIF plays a role in cilia biogenesis and directly regulates the transcription of genes associated with cell biology and function remains elusive. In this study, we present for the first time that MIF 1) is a novel centriole protein which forms a ring like structure at proximal end of centrioles to regulate cilia biogenesis, and 2) is a novel transcriptional factor to regulate the expression of genes related to ciliogenesis and other signaling pathways. In addition, we identified that PIP4K2a is an upstream regulator of MIF, which phosphorylates MIF at S91 to promote the interaction of MIF with 14-3-3ろ and its nuclear translocation (Fig. 8).

Cilia biogenesis is a multistep process in which the mother centriole plays a fundamental role. Upon cell cycle exit, this older centriole associates with a Golgi-derived ciliary vesicle at its distal end. After fusion of additional vesicles, the mother centriole migrates to the cell surface, where the centriole-associated vesicles fuse with the plasma membrane. The mother centriole-to-basal body transition and the extension of the ciliary axoneme involve prominent changes in protein composition [34]. However, whether and how an inflammatory cytokine cooperates with other factors to spatial-temporally regulate cilia biogenesis remains unknown. The first key feature of this study is to identify MIF as a proximal end protein of centrioles to govern centriolar structure and control a balance between ciliary assembly and disassembly. As a component of centrioles, MIF regulates the removal of CP110 from mother centriole and the recruitment of TTBK2 to basal body (Supplementary Fig. 5), the accumulation of CEP290 and BBS4 in the centriolar satellites (Supplementary Fig. 6), the translocation of HYLS-1 from basal body into axoneme (Fig. 4), and the trafficking and entry of IFT and IFT related proteins to axoneme (Supplementary Fig. 7, 8).

CP110 has been recognized as a crucial and negative regulator of cilia biogenesis. CP110 is localized on the distal ends of both mother and daughter centrioles, which forms a cap to block the elongation of axonemal microtubules and, thereby, prevents inappropriate ciliogenesis in proliferating cells [23]. CP110 is induced as cells enter $\mathrm{S}$ phase and downregulated at G2/M and G1 phase. In response to cell cycle exit signals, CP110 is removed selectively from the mother centriole and degraded through ubiquitylation, which is an essential event for the quiescence-induced ciliogenesis [35]. However, the detailed mechanisms underlying this event are still unclear. We found that MIF is required for CP110 to the distal ends of the mother centrioles, which may function as a switch for initiating the early steps of ciliogenesis. Loss of MIF perturbs centriolar localization of CP110 in mammalian cells (Supplementary Fig. 5a-d). The removal of CP110 coincides with the presence of TTBK2 at mother centriole, which is also one of the earliest identified events in ciliogenesis [29]. TTBK2 was physically associated with microtubules, which could phosphorylate microtubule-associated proteins in vitro [29]. TTBK2 promotes the removal of CP110 and promotes the recruitment of IFT proteins, which build the ciliary axoneme. We found that depletion of MIF increased the recruitment of TTBK2 to basal body (Supplementary Fig. $5 \mathrm{i}$, j), suggesting that MIF is an upstream regulator of TTBK2-CP110 signaling and plays an important role in TTBK2-CP110 mediated 
ciliary initiation. Whether MIF regulates microtubule dynamics via TTBK2 during the cell cycle, however, still needs to be addressed.

Centriolar satellites, which are no membranous, electron-dense particles surrounding centrosomes in mammalian cells, play important roles during cilium assembly and function [22]. CEP290 and BBS4 are localized at centriolar satellites, which function to regulate the trafficking of ciliary proteins into and out of the cilium [26, 30]. CEP290 interacts with and inhibits CP110 to suppress ciliogenesis [25]. We found that depletion of MIF increased the accumulation of CEP290 at centriolar satellite (Supplementary Fig. 6a, b), suggesting a functional network among MIF, CEP290 and CP110. Centriolar satellites could sequester BBS4 and regulate recruitment of the BBSome complex to the cilium by limiting the amount of BBS4 available for incorporation into ciliary BBSomes [36]. We found that depletion of MIF increased the accumulation of BBS4 at centriolar satellite and its ciliary entry (Supplementary Fig. 6c, d), suggesting a role of MIF in the assembly of BBS4 into the BBSome complex and its subsequent ciliary recruitment.

HYLS-1 has been identified as a component of outer centriole wall in C. elegans [37]. Loss of HYLS-1 compromises the docking and entry of IFT particles, ciliary gating for both membrane and soluble proteins, and axoneme assembly [27]. The fly HYLS-1 is required for efficient pericentriolar material (PCM) components recruitment in spermatocyte and the proximal centriole-like structure (PCL) formation in spermatids [38]. Mutation of HYLS-1 results in hydrolethalus syndrome, which is a rare recessive lethal inherited disorder that causes serious fetal development and birth defects [38]. We found that MIF interacted with HYLS-1 (Fig. 4j-m), which prevented the translocation of HYLS-1 from basal body into the axoneme in mammalian cells (Fig. 4o-r). The interaction of MIF with HYSL-1 further supports that MIF is a centriolar protein. In addition, these results indicated for the first time that HYLS-1 could enter the axoneme and MIF might gate this process, and also suggested that MIF might be involved in fetal development and hydrolethalus syndrome.

IFT proteins are critical for the bidirectional movement of multiprotein complexes along the axoneme, and two families of molecular motors, kinesins and cytoplasmic dyneins, are involved in these processes. IFT proteins organize into two complexes, IFT-A and IFT-B. IFT-A proteins regulate retrograde trafficking of ciliary cargos, whereas IFT-B proteins mediate anterograde transport of ciliary cargos from the cell body to the cilium tip. Kinesin-II is responsible for transport of the raft complex and the components used for cilia construction and maintenance to the tip of cilia [39]. Conditional knockout of KIF3a resulted in a loss of cilia, demonstrating that KIF3a is necessary for ciliogenesis [40]. IFT20 is the smallest IFT protein in the IFT-B complex. In living cells, fluorescently tagged IFT20 is highly dynamic and moves between the Golgi complex and the cilium as well as along ciliary microtubules [41], which functions in the delivery of ciliary membrane proteins from Golgi to cilium. Knockdown of IFT20 blocks ciliary assembly but does not affect Golgi structure in mammalian cells [41]. We found that depletion of MIF induced an accumulation of KIF3a and IFT20 at cilia tips (Supplementary Fig. 7, 8). In addition, depletion of MIF also resulted in an accumulation of IFT20 around the basal body (Supplementary Fig. 7, 8). Interestingly, we also found that depletion of MIF increased the amount of IFT88 along the cilia (Supplementary Fig. 7, 8), but decreased the tip location of IFT140 (Supplementary Fig. 7, 8). Our results suggest that loss of MIF may increase the 
trafficking of ciliary proteins from Golgi to the base of cilia and their trafficking along cilia. The role of MIF in regulation of the trafficking of other IFTs needs to be further investigated.

The second key feature of this study is to identify MIF as a transcriptional factor. MIF directly binds on the promoters of genes related to ciliogenesis, including KIF3a, IFT20, CEP290 and BBS4 (Supplementary Fig. 11). ChIP-seq analysis suggested that MIF also regulates the transcription of a broad network of ciliary related genes including those gene encoding the components of IFT A particles, IFT B particles, transition zone, distal appendage, axoneme, basal body, centriolar satellite, and BBSome, etc. (Fig. 8b). These results further support a role and mechanism of MIF in regulation of cilia biogenesis. MIF has been reported to regulate diverse signaling pathways mainly via binding on its membrane receptors [7]. However, whether and how MIF can directly regulate intracellular signaling pathways remains unknown. We found that MIF also targets genes associated with diverse signaling pathways that control spliceosome, RNA transport, cell cycle, apoptosis, proliferation, ribosome, autophagy, etc., and genes associated with Parkinson disease, Huntington disease, renal cell carcinoma and viral carcinogenesis (Fig. 5), suggesting that MIF should play an important role in homeostasis and disease progression via directly regulating the expression of genes in those signaling pathways and diseases. In particular, the MIF target genes in cell cycle regulation provide another potential mechanism to support our finding that MIF is involved in the regulation of cystic renal epithelial cell proliferation, leading to cyst growth in kidneys [6]. These results should forward our understanding of the roles and mechanisms of MIF in human diseases.

The third key feature of this study is to identify PIP4K2a as an upstream regulator of MIF. The activity of MIF can be regulated with its phosphorylation [42]. With the analyses of protein chip as well as immunoprecipitation and mass spectrometry (MS) we identified several kinases, including PIP4K2a, PRKD2, TLK1 and BRAF, that may be responsible for MIF phosphorylation. It has been reported that PIP4K2a facilitates the conversion of phosphorylation of phosphatidylinositol 5-phosphate (Ptdlns5P) to generate various phosphoinositide species, which regulate crucial cellular functions including proliferation, survival, glucose uptake and migration [43, 44]. Depletion of PIP4K2a is associated with a decrease of mTOR signaling, and induces developmental defects in Drosophila and zebrafish [43]. We confirmed a interaction between MIF and PIP4K2a (Fig. 7a, b), and found that PIP4K2a phosphorylated MIF at S91 (Fig. 7). We also found that wild type MIF and a MIF S91D phosphomimetic mutant could enter nucleus, whereas a non-phosphorylatable MIF S91A mutant failed to be translocated into the nucleus (Fig. 6). In addition, phosphorylation of MIF increased its interaction with 14-3-3ろ (Fig. 6), which facilitated the nuclear translocation of MIF. These results provide a mechanism of how MIF is shuttled from cytoplasm to nucleus where MIF functions as a transcriptional factor. In addition, we found that knockdown or overexpression of PIP4K2a also resulted in an increase or decrease of ciliated cells and cilia length, respectively (Fig. 7f-m). These results suggest that PIP4K2a is an upstream regulator of MIF activity, which should also regulate cilia biogenesis, proliferation, survival, glucose uptake and migration via MIF target genes. 
In sum, this study demonstrates for the first time that an inflammatory cytokine, MIF, is a centriolar structure protein to directly regulate cilia biogenesis, suggesting a crosstalk of inflammation and ciliopathies. This study also identifies MIF as a novel transcriptional factor, which forwards our understanding of the roles and mechanisms of MIF in homeostasis and human diseases. In addition, this study identifies that a kinase, PIP4K2a, as an upstream regulator of MIF activity, which phosphorylates MIF at S91 to promote the interaction of MIF with 14-3-3 and MIF nuclear translocation.

\section{Methods}

Cell culture and reagents. Human renal cortical tubular epithelia (RCTE) and hTERT-immortalized retinal pigment epithelial cell (hTERT-RPE-1) were maintained at $37^{\circ} \mathrm{C}$ in $5 \% \mathrm{CO}_{2}$ in DMEM/F12 containing $10 \%$ fetal bovine serum, supplemented with penicillin and streptomycin. Hela cells and HEK293T cells were purchased from ATCC (Manassas, VA, USA) and cultured in DMEM containing 10\% fetal bovine serum, supplemented with penicillin and streptomycin.

For plasmid transfection, Lipofectamine 3000 (Thermo Fisher) was used, according to the manufacturer's instructions. For siRNAs transfection, Lipofectamine RNAiMAX (Invitrogene) was used, according to the manufacturer's instructions.

Nocodazole and thymidine were purchased from Sigma. ISO-1 was purchased from EMD Millipore. Recombinant human MIF was purchased from R\&D Systems. Lambda phosphatase was purchased from Santa Cruz.

Mouse primary tubular cells isolation. After removal of renal capsules and medulla, kidney sections were minced into tiny pieces, and incubated in $10 \mathrm{~mL}$ of a $1 \%$ collagenase type I buffer in a $37^{\circ} \mathrm{C}$ oven with gentle rotation for $30 \mathrm{~min}$. Then undigested kidney tissues were removed through a $70 \mu \mathrm{m}$ filter. The filtered cell suspension was centrifuged at $150 \mathrm{~g}$ for $5 \mathrm{~min}$. The pellet was washed with PBS and then centrifuged and resuspended and cultured in DME/ F12 containing $2 \%$ fetal bovine serum, supplemented with penicillin and streptomycin, $10 \mathrm{ng} / \mathrm{mL}$ of epidermal growth factor, $5 \mu \mathrm{g} / \mathrm{mL}$ of insulin, $0.5 \mu \mathrm{g} / \mathrm{mL}$ of epinephrine, $36 \mathrm{ng} / \mathrm{mL}$ of hydrocortisone, $5 \mu \mathrm{g} / \mathrm{mL}$ of transferrin, and $4 \mathrm{pg} / \mathrm{mL}$ of triiodo-L-thyronine).

Mice. Mif whole body knockout (KO) mice were fertile [45]. Mif ${ }^{-1-}$ mice were on the C57BL/6 genetic background. All animal experiments were conducted under the IACUC protocol: A00003756-18, which was reviewed and approved by the IACUC of the Mayo Clinic, in accordance with the National Institutes of Health, United States Department of Agriculture, and the Association for Assessment and Accreditation of Laboratory Animal Care guidelines.

Antibodies, drugs and reagents. The antibodies used for Western blot analysis included, (a) anti-MIF (ab36136) antibody which was purchased from Abcam; (b) anti-PIP4K2a (sc-100406), anti-14-3-3Z (sc293415), anti-Cyclin B (sc-4135), anti-CDC20 (sc-14866), anti-CDH1 (sc-56312), anti-HYLS-1(sc-376721), anti-GFP (sc-9996) antibodies which were purchased from Santa Cruz Biotechnology Inc; (c) anti-T/S pan (\# 9631), anti-Lamin A/C (\# 2032) antibodies which were purchased from Cell Signaling Technology; (d) 
anti-KIF3a (13930-1-AP), anti-IFT20 (13615-1-AP), anti-IFT88 (13967-1-AP), anti-IFT140 (17460-1-AP) antibodies which were purchased from Proteintech; (e) anti- $\beta$-actin antibody (A5316) antibody which was purchased from Sigma-Aldrich. The secondary antibodies, including donkey anti-rabbit IgG-horseradish peroxidase (sc-2313) and goat anti-mouse IgG-horseradish peroxidase (sc-2005), were purchased from Santa Cruz Biotechnology, Inc. The antibodies used for Immunofluorescent staining included, (a) anti-MIF (ab36136), anti-MIF (sc-20121), anti-CEP135 (ab75005), anti-CEP290 (ab84870) antibodies which were purchased from Abcam, (b) anti-GFP (sc-9996), anti-CEP164 (sc-515403), anti-Ninein (sc-376420), anti-cNap1 (sc-390540), anti-HYLS-1(sc-376721), anti-PIP4K2a (sc-100406) antibodies which were purchased from Santa Cruz Biotechnology Inc; (c) anti-gamma-tubulin (T5326), anti-acetylated a-tubulin (T7451), anti-TTBK2 (HPA018113) antibodies which were purchased from Sigma-Aldrich; (d) anti-CP110 (127801AP), anti-BBS4 (12766-1-AP), anti-KIF3a (13930-1-AP), anti-IFT20 (13615-1-AP), anti-IFT88 (13967-1-AP), anti-IFT140 (17460-1-AP) antibodies which were purchased from Proteintech; (e) anti-pericentrin (ABT59) antibody which was purchased from EMD Millipore. The Alexa Fluor secondary antibody 555 and 488 were purchased from Thermo Fisher.

DNA constructs and siRNAs. The GFP-tagged MIF (RG205106) and GFP-tagged PIP4K2a (RG205243) plasmids were purchased from Origene. The mutant MIF plasmids, including the S91D and S91A mutants were generated by KOD-Plus-Mutagenesis Kit (TOYOBO) following the manufacturer's instructions. The RNA oligonucleotides that specifically target human MIF (sc-37137), human PIP4K2a (sc-39139), human 14-3-3ろ (sc-29583) were purchased from Santa Cruz Biotechnology, Inc.

\section{RNA extraction and quantitative reverse transcription-polymerase chain reaction (qRT-PCR). Total RNA} was extracted using the RNeasy Plus Mini Kit (QIAGEN). Total RNA $(1 \mu \mathrm{g})$ was used for reverse transcription reactions in a $20 \mu$ reaction to synthesize cDNA with an iScript cDNA Synthesis Kit (BioRad). RNA expression profiles were analyzed by real-time PCR using iTaq SYBR Green Supermix with ROX (Bio-Rad) in an iCycler iQ Real-Time PCR Detection System. The complete reactions were subjected to the following program of thermal cycling: 40 cycles of $10 \mathrm{~s}$ at $95^{\circ} \mathrm{C}$ and $20 \mathrm{~s}$ at $60^{\circ} \mathrm{C}$. A melting curve was run after the PCR cycles followed by a cooling step. Each sample was run in triplicate in each experiment, and each experiment was repeated 3 times. Expression levels of target genes were normalized to the expression level of actin. All primers used are listed in Supplementary Table 1.

Protein extraction and Western blot analysis. Cell pellets were collected and resuspended in lysis buffer (20 mM Tris-HCl, pH 7.4, 150 mM NaCl, 10\% glycerol, 1\% Triton X-100, 1 mM Na3VO4, 25 mM $\beta$ glycerolphosphate, $0.1 \mathrm{mM}$ PMSF, Roche complete protease inhibitor set, and Sigma-Aldrich phosphatase inhibitor set). The resuspended cell pellet was vortexed for $20 \mathrm{~s}$ and then incubated on ice for $30 \mathrm{~min}$ and centrifuged at 20,000 g for $30 \mathrm{~min}$. The supernatants were collected for Western blot analysis or immunoprecipitation.

For immunoprecipitation, anti-MIF or anti-GFP or anti-PIP4K2a antibodies and their isotype control antibodies were coupled to protein A agarose beads (Pierce) in PBS containing $5 \mathrm{mg} / \mathrm{ml}$ bovine serum albumin (Sigma-Aldrich) for $6 \mathrm{~h}$ at $4^{\circ} \mathrm{C}$ on a rotating platform. The cell lysates were then incubated with 
the beads coupled with antibodies at $4^{\circ} \mathrm{C}$ overnight. Then the beads were washed with lysis buffer containing an additional $300 \mathrm{mM} \mathrm{NaCl}$, and the immune complexes were eluted off the beads using loading buffer with boiling for $5 \mathrm{~min}$ and then subjected to Western blot analysis.

Immunofluorescence microscopy. Briefly, cells on coverslips were fixed in pre-cold methanol for $10 \mathrm{~min}$ at $-20^{\circ} \mathrm{C}$, followed by permeabilization with $0.1 \%$ Triton X-100 for $15 \mathrm{~min}$ at room temperature. After blocking in $2 \%$ BSA, cells were incubated with appropriate primary and secondary antibodies. For tissue staining, mouse kidneys were dissected out from 21 days-old or 3 months-old mice, fixed in 4\% PFA in PBS at room temperature overnight, further dehydrated and infiltrated the tissue with paraffin, then embed the tissue in paraffin,

Images were acquired using a Zeiss confocal microscope LSM780 or a Nikon Eclipse 80i microscope. Three-dimensional structured illumination microscopy experiments were performed using a Zeiss ELYRA super-resolution microscopy system using $63 \times 1.4$ oil immersion lens and five phases and three rotations of the illumination pattern. Images were processed by 3D transparency-rendering method.

Extraction of cytoplasmic and nuclear proteins. The cytosol extracts (C) and nuclear extracts $(\mathrm{N})$ are the fraction prepared from whole-cell lysates. Briefly, cell pellets were collected and resuspended in cytoplasmic extract buffer (10 mM HEPES pH 7.9, $10 \mathrm{mM} \mathrm{KCl,} 0.1 \mathrm{mM}$ EDTA, 0.3\% NP-40, protease inhibitors $1 \mathrm{x}$ ). The resuspended cell pellet was vortexed for $5 \mathrm{~min}$ on ice and then centrifuged at 3000 $\mathrm{rpm}$ for $5 \mathrm{~min}$ at $4^{\circ} \mathrm{C}$. The supernatants were harvested as the cytoplasmic extracts. Resuspended and washed the pellet in cytoplasmic extract buffer without NP-40 and centrifuged at $3000 \mathrm{rpm}$ for $5 \mathrm{~min}$. Then resuspended the pellet in nuclear extract buffer $(20 \mathrm{mM}$ HEPES pH 7.9, 0.4 M NaCl, 1 mM EDTA, $25 \%$ Glycerol, protease inhibitors $1 \mathrm{x}$ ) and incubated on ice for $10 \mathrm{~min}$. Centrifuged at 14,000 rpm for 5 min at $4^{\circ} \mathrm{C}$ and harvested supernatant (nuclear extract). The samples were used for Western blot analysis.

Cell synchronization and cell cycle analyses. RCTE and hTERT-RPE-1 cells were synchronized by serum deprivation and restimulation as described $[23,35]$. RCTE cells and Hela cells were also synchronized with thymidine followed by nocodazole for $\mathrm{G} 2 / \mathrm{M}$ arrest and double thymidine for $\mathrm{G} 1 / \mathrm{S}$ arrest.

For G2/M arrest, RCTE and Hela cells were subjected to thymidine ( $2 \mathrm{mM}$, Sigma-Aldrich) for $18 \mathrm{~h}$, followed by release for $6 \mathrm{~h}$, and subsequently blocked again with nocodazole ( $250 \mathrm{ng} / \mathrm{mL}$, Sigma-Aldrich) for $12 \mathrm{~h}$. For G1/S arrest, cells were subjected to thymidine ( $2 \mathrm{mM}$, Sigma-Aldrich) for $18 \mathrm{~h}$, followed by release for $12 \mathrm{~h}$, and subsequently blocked again with thymidine for $18 \mathrm{~h}$. Propidium iodide staining and FACS analysis were performed as described [23]. Briefly, 70\% ethanol-fixed cells were treated with 20 $\mu \mathrm{g} / \mathrm{ml}$ RNase $A$ and $10 \mu \mathrm{g} / \mathrm{ml}$ propidium iodide for $30 \mathrm{~min}$ before being subjected to cell cycle analyses with FACS Calibur flow cytometry (BD Biosciences).

ChIP assay. ChIP assays were performed according to the protocol described previously [29]. Chromatin DNA was subjected to immunoprecipitation with anti-MIF, anti-H3 antibody, or normal goat IgG, and then washed, after which the DNA-protein crosslinks were reversed. Negative control primers were located 
about 2000 bp upstream of the transcription start site (TSS) of targeted genes. The recovered DNA was analyzed by PCR for the binding of MIF at the respective human ciliary gene promoters.

ChIP-seq analysis. The anti-MIF antibody (ab36146, Abcam) was used and DNA was immunoprecipitated from the sonicated cell lysates. Each sample with $10 \mathrm{ng}$ of DNA generated from the ChIP assay was used to construct the sequencing libraries with the ChIP-Seq Sample Prep Kit (Illumina). Raw data of ChIP-seq was analyzed as previously described [46].

Human protein chip high-throughput screening. Human protein chips which were prepared by spotting more than 21,000 highly purified proteins onto special nitrocellulose-coated slides, were incubated in renaturation buffer containing $50 \mathrm{mM}$ Tris-HCl, pH 8.0, $100 \mathrm{mM} \mathrm{NaCl}, 1 \mathrm{mM} \mathrm{DTT}, 0.3 \%$ Tween 20 for $1 \mathrm{~h}$ at $4^{\circ} \mathrm{C}$. After Blocking with $5 \%$ bovine serum albumin for $1 \mathrm{~h}$ at room temperature, protein chips were incubated with purified human MIF protein ( $50 \mathrm{nM}$, R\&D Systems) or $5 \%$ bovine serum albumin for 1 hour. Protein interaction was then determined by sequentially incubating with either rabbit anti-MIF antibody and goat anti-rabbit immunoglobulin $\mathrm{G}(\mathrm{IgG})$-Cy3-conjugated antibody or goat anti-rabbit immunoglobulin G (IgG)-Cy3-conjugated antibody (negative controls). Axon GenePix 4000B Microarray Scanner (Molecular Devices, LLC, Sunnyvale, CA), and the probe signals were acquired using GenePix Pro 6.0 software (Molecular Devices). The probes were considered detectable when the signal-to-noise ratios (SNRs) for both duplicates were over 1.2 as described previously [47].

Co-IP and LC-MS/MS analysis. The procedure for LC-MS/MS was performed as described previously [48]. The lysates of RCTE cells were collected and immunoprecipitated with MIF antibody. Then the Immunoprecipitation samples were separated by sodium dodecyl sulfate (SDS)-polyacrylamide gel electrophoresis, and visualized with colloidal Coomassie blue staining. Protein bands visible after coomassie blue staining were subjected to in-gel reduction, carboxyamidomethylation and tryptic digestion. Digested peptides were measured on a Dionex Ultimate 3000 nano-LC system coupled to a linear quadrupole ion trap-Orbitrap mass spectrometer equipped with a nanoelectrospray ion source 16. Protein identification was performed by searching the data against the databases using Mascot Deamon. Subcellular location of the identified proteins was determined using DAVID bioinformatics resources.

Statistical analysis. Statistical significance was determined by (multiple) student's t-test or one-way ANOVA. Data were presented as mean \pm SD or SEM. Differences with $p$ values less than 0.05 were considered statistically significant. Experiments for quantification were performed in a blinded fashion. In order to ensure adequate power to detect the effect, at least 3 independent tests were performed.

\section{Data availability statement}

Protein chip and Mass spectrometry data will be deposited to the EMBL-EBI MetaboLights database. All other data supporting the findings of this study are available from the corresponding author upon reasonable request. Source data are provided with this paper.

\section{Declarations}




\section{Competing interests}

The authors have declared that no competing interest exists.

\section{Author contributions}

L.Z. performed most experiments and data analysis. H. Z. and L.X.L. and E.A. performed some of the experiments and data analysis. X.L. supervised the whole project, data analysis and manuscript writing.

\section{Acknowledgements}

We are grateful to Jinghua Hu (Mayo Clinic) for providing cell lines RCTE and RPE cells.

Funding: X. Li acknowledges support from National Institutes of Health grant R01 DK084097, R01 DK126662, and NIH P30 DK106912 and from the PKD Foundation.

\section{References}

1.Calandra, T. and T. Roger, Macrophage migration inhibitory factor: a regulator of innate immunity. Nat Rev Immunol, 2003. 3(10): p. 791-800.

2.Sanchez-Nino, M.D., et al., MIF, CD74 and other partners in kidney disease: tales of a promiscuous couple. Cytokine Growth Factor Rev, 2013. 24(1): p. 23-40.

3.Leng, L., et al., MIF signal transduction initiated by binding to CD74. J Exp Med, 2003. 197(11): p. 146776.

4.Bernhagen, J., et al., MIF is a noncognate ligand of CXC chemokine receptors in inflammatory and atherogenic cell recruitment. Nat Med, 2007. 13(5): p. 587-96.

5.Wilson, J.M., et al., Macrophage migration inhibitory factor promotes intestinal tumorigenesis. Gastroenterology, 2005. 129(5): p. 1485-503.

6.Chen, L., et al., Macrophage migration inhibitory factor promotes cyst growth in polycystic kidney disease. J Clin Invest, 2015. 125(6): p. 2399-412.

7.Fukaya, R., et al., MIF Maintains the Tumorigenic Capacity of Brain Tumor-Initiating Cells by Directly Inhibiting p53. Cancer Res, 2016. 76(9): p. 2813-23.

8.Kleemann, R., et al., Intracellular action of the cytokine MIF to modulate AP-1 activity and the cell cycle through Jab1. Nature, 2000. 408(6809): p. 211-6.

9.Talos, F., et al., MIF loss impairs Myc-induced lymphomagenesis. Cell Death Differ, 2005. 12(10): p. 1319-28. 
10.Fan, J., et al., Removing intensity effects and identifying significant genes for Affymetrix arrays in macrophage migration inhibitory factor-suppressed neuroblastoma cells. Proc Natl Acad Sci U S A, 2005. 102(49): p. 17751-6.

11.Fingerle-Rowson, G., et al., The p53-dependent effects of macrophage migration inhibitory factor revealed by gene targeting. Proc Natl Acad Sci U S A, 2003. 100(16): p. 9354-9.

12.Liu, L., et al., A global genomic view of MIF knockdown-mediated cell cycle arrest. Cell Cycle, 2008. 7(11): p. 1678-92.

13.Plotnikova, O.V., E.N. Pugacheva, and E.A. Golemis, Primary cilia and the cell cycle. Methods Cell Biol, 2009. 94: p. 137-60.

14.Sorokin, S., Centrioles and the formation of rudimentary cilia by fibroblasts and smooth muscle cells. J Cell Biol, 1962. 15: p. 363-77.

15.Nigg, E.A. and T. Stearns, The centrosome cycle: Centriole biogenesis, duplication and inherent asymmetries. Nat Cell Biol, 2011. 13(10): p. 1154-60.

16.Sun, S., et al., Three-dimensional architecture of epithelial primary cilia. Proc Natl Acad Sci U S A, 2019. 116(19): p. 9370-9379.

17.Singla, V. and J.F. Reiter, The primary cilium as the cell's antenna: signaling at a sensory organelle. Science, 2006. 313(5787): p. 629-33.

18.Brueckner, M., Heterotaxia, congenital heart disease, and primary ciliary dyskinesia. Circulation, 2007. 115(22): p. 2793-5.

19.Sanchez, I. and B.D. Dynlacht, Cilium assembly and disassembly. Nat Cell Biol, 2016. 18(7): p. 711-7.

20.Graser, S., et al., Cep164, a novel centriole appendage protein required for primary cilium formation. J Cell Biol, 2007. 179(2): p. 321-30.

21.Fry, A.M., et al., C-Nap1, a novel centrosomal coiled-coil protein and candidate substrate of the cell cycle-regulated protein kinase Nek2. J Cell Biol, 1998. 141(7): p. 1563-74.

22.Breslow, D.K. and A.J. Holland, Mechanism and Regulation of Centriole and Cilium Biogenesis. Annu Rev Biochem, 2019. 88: p. 691-724.

23.Kobayashi, T., et al., Centriolar kinesin Kif24 interacts with CP110 to remodel microtubules and regulate ciliogenesis. Cell, 2011. 145(6): p. 914-25.

24.Nagai, T., et al., Cullin-3-KCTD10-mediated CEP97 degradation promotes primary cilium formation. J Cell Sci, 2018. 131(24). 
25.Tsang, W.Y., et al., CP110 suppresses primary cilia formation through its interaction with CEP290, a protein deficient in human ciliary disease. Dev Cell, 2008. 15(2): p. 187-97.

26.Stowe, T.R., et al., The centriolar satellite proteins Cep72 and Cep290 interact and are required for recruitment of BBS proteins to the cilium. Mol Biol Cell, 2012. 23(17): p. 3322-35.

27.Wei, Q., et al., The hydrolethalus syndrome protein HYLS-1 regulates formation of the ciliary gate. Nat Commun, 2016. 7: p. 12437.

28.Inanc, B., et al., Abnormal centrosomal structure and duplication in Cep135-deficient vertebrate cells. Mol Biol Cell, 2013. 24(17): p. 2645-54.

29.Goetz, S.C., K.F. Liem, Jr., and K.V. Anderson, The spinocerebellar ataxia-associated gene Tau tubulin kinase 2 controls the initiation of ciliogenesis. Cell, 2012. 151(4): p. 847-858.

30.Uytingco, C.R., et al., BBS4 is required for intraflagellar transport coordination and basal body number in mammalian olfactory cilia. J Cell Sci, 2019. 132(5).

31.Kodani, A., et al., Kif3a interacts with Dynactin subunit p150 Glued to organize centriole subdistal appendages. EMBO J, 2013. 32(4): p. 597-607.

32.Morrison, D.K., The 14-3-3 proteins: integrators of diverse signaling cues that impact cell fate and cancer development. Trends Cell Biol, 2009. 19(1): p. 16-23.

33.Sakiyama, H., et al., Regulation of nuclear import/export of carbohydrate response element-binding protein (ChREBP): interaction of an alpha-helix of ChREBP with the 14-3-3 proteins and regulation by phosphorylation. J Biol Chem, 2008. 283(36): p. 24899-908.

34.Kim, S. and B.D. Dynlacht, Assembling a primary cilium. Curr Opin Cell Biol, 2013. 25(4): p. 506-11.

35.Huang, N., et al., M-Phase Phosphoprotein 9 regulates ciliogenesis by modulating CP110-CEP97 complex localization at the mother centriole. Nat Commun, 2018. 9(1): p. 4511.

36.Conkar, D., et al., The centriolar satellite protein CCDC66 interacts with CEP290 and functions in cilium formation and trafficking. J Cell Sci, 2017. 130(8): p. 1450-1462.

37.Dammermann, A., et al., The hydrolethalus syndrome protein HYLS-1 links core centriole structure to cilia formation. Genes Dev, 2009. 23(17): p. 2046-59.

38.Hou, Y., et al., Functional Analysis of Hydrolethalus Syndrome Protein HYLS1 in Ciliogenesis and Spermatogenesis in Drosophila. Front Cell Dev Biol, 2020. 8: p. 301.

39.Bisgrove, B.W. and H.J. Yost, The roles of cilia in developmental disorders and disease. Development, 2006. 133(21): p. 4131-43. 
40.Pooranachandran, N. and J.J. Malicki, Unexpected Roles for Ciliary Kinesins and Intraflagellar Transport Proteins. Genetics, 2016. 203(2): p. 771-85.

41.Follit, J.A., et al., The intraflagellar transport protein IFT20 is associated with the Golgi complex and is required for cilia assembly. Mol Biol Cell, 2006. 17(9): p. 3781-92.

42.Watarai, H., et al., Posttranslational modification of the glycosylation inhibiting factor (GIF) gene product generates bioactive GIF. Proc Natl Acad Sci U S A, 2000. 97(24): p. 13251-6.

43.Gupta, A., et al., Phosphatidylinositol 5-phosphate 4-kinase (PIP4K) regulates TOR signaling and cell growth during Drosophila development. Proc Natl Acad Sci U S A, 2013. 110(15): p. 5963-8.

44.Kitagawa, M., et al., Dual blockade of the lipid kinase PIP4Ks and mitotic pathways leads to cancerselective lethality. Nat Commun, 2017. 8(1): p. 2200.

45.Gadjeva, M., et al., Inhibition of macrophage migration inhibitory factor ameliorates ocular Pseudomonas aeruginosa-induced keratitis. PLoS Pathog, 2010. 6(3): p. e1000826.

46.Li, L.X., et al., Lysine methyltransferase SMYD2 promotes cyst growth in autosomal dominant polycystic kidney disease. J Clin Invest, 2017. 127(7): p. 2751-2764.

47.Fan, Q., et al., Identification of proteins that interact with alpha A-crystallin using a human proteome microarray. Mol Vis, 2014. 20: p. 117-24.

48. Han, Y., et al., Proteomic investigation of the interactome of FMNL 1 in hematopoietic cells unveils a role in calcium-dependent membrane plasticity. J Proteomics, 2013. 78: p. 72-82.

\section{Figures}


a

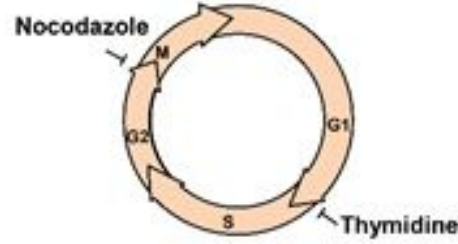

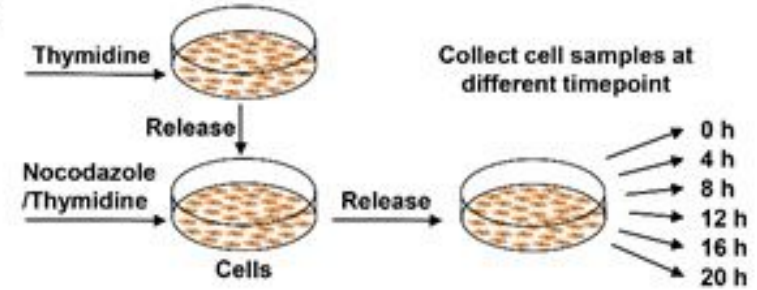
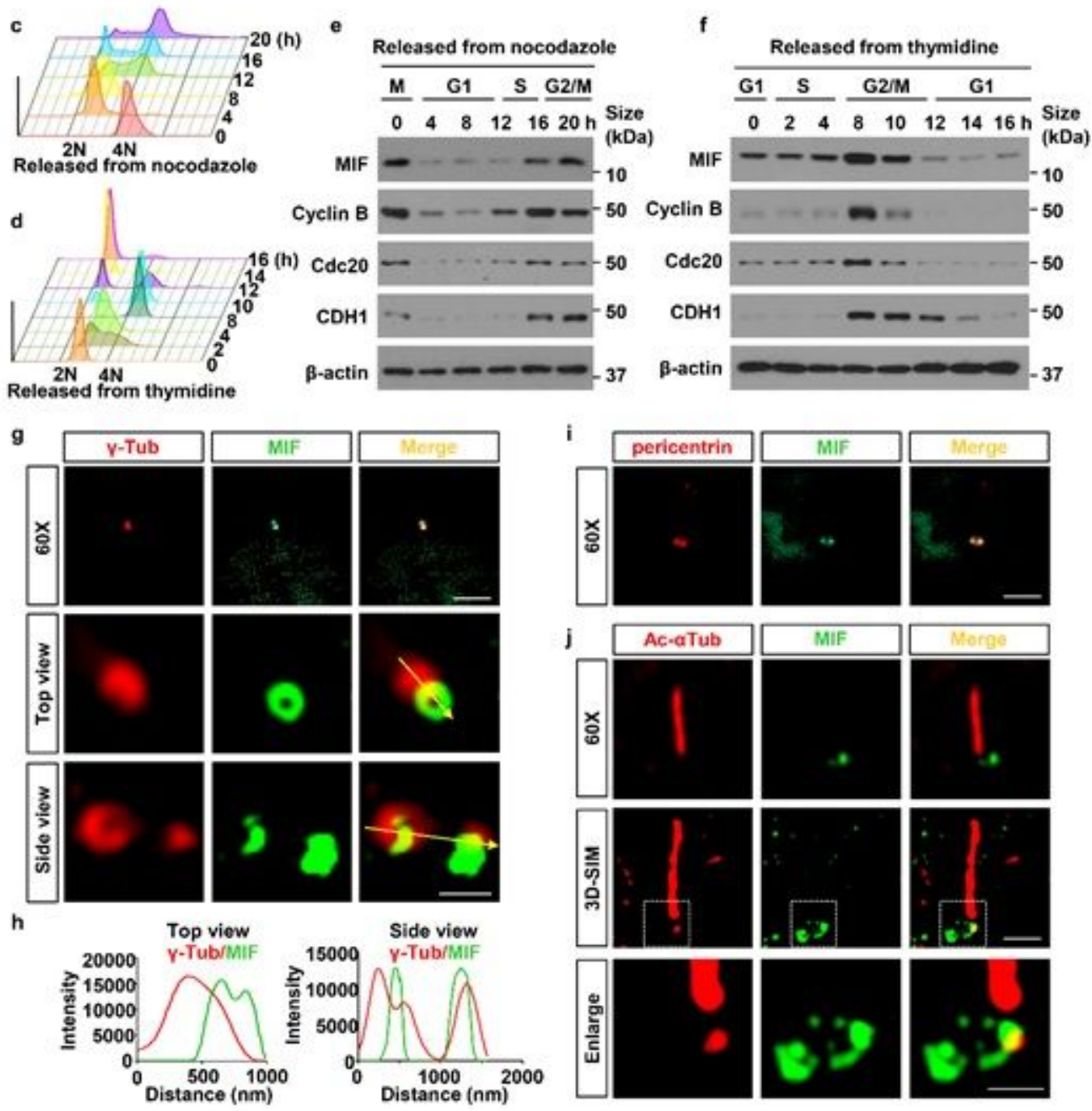

\section{Figure 1}

The levels of MIF protein fluctuate throughout cell cycle and MIF is located at basal body in RCTE cells. a Schematic showing the cell cycle phase inhibited by nocodazole and thymidine. b Experimental outlines for using thymidine-nocodazole block or double thymidine block methods to synchronize RCTE cells. c, d Fluorescence-activated cell sorting (FACS) was used to estimate the cell-cycle profiles in thymidine/nocodazole (c) and double thymidine block (d) treated cells by measuring the DNA contents in those cells with propidium iodide staining. $\mathrm{e}, \mathrm{f}$ Western blot analysis of whole cell lysates derived from RCTE cells synchronized in M phase by thymidine-nocodazole (e) and in late G1/S phase by double thymidine treatment (f) following by releasing back into the cell cycle. $g$ Representative images of RCTE cells stained with MIF (green) antibody and co-stained with $\mathrm{y}$-tubulin ( $\mathrm{\gamma}$-Tub) (red), which were visualized under 2D microscope and three-dimensional structured illumination microscopy (3D-SIM). Scale bars, 5 
$\mu \mathrm{m}$ (top panels) and $500 \mathrm{~nm}$ (middle and bottom panels of 3D images). $\mathrm{h}$ The intensity plots of the rings in 3D images (g) from top and side views. I Representative images of RCTE cells stained with MIF (green) antibody and co-stained with pericentrin (red). Scale bar, $5 \mu \mathrm{m}$. j Representative images of RCTE cells stained with MIF (green) antibody and co-stained with acetylated a-tubulin (Ac-a-Tub) (red), which were visualized under 2D microscope and 3D-SIM microscopy. Scale bars, $5 \mu \mathrm{m}$ (top panels) and $500 \mathrm{~nm}$ (middle and bottom panels of 3D images).
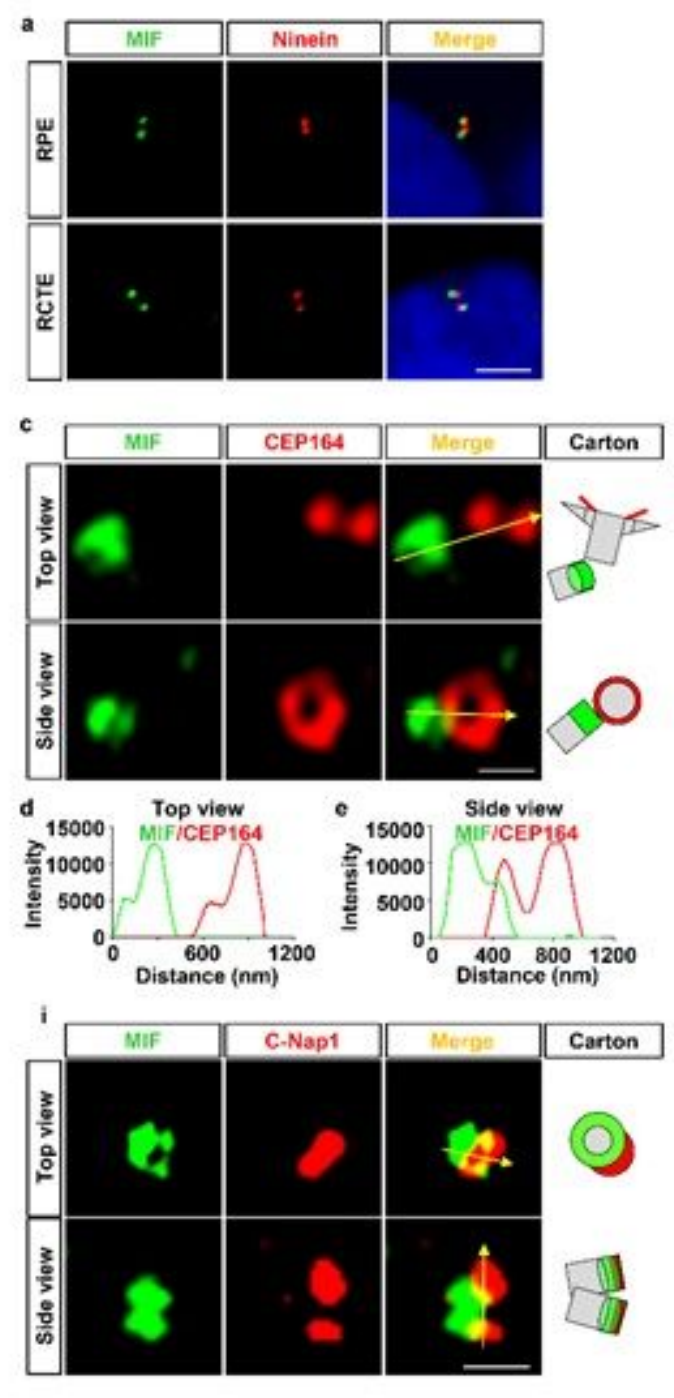
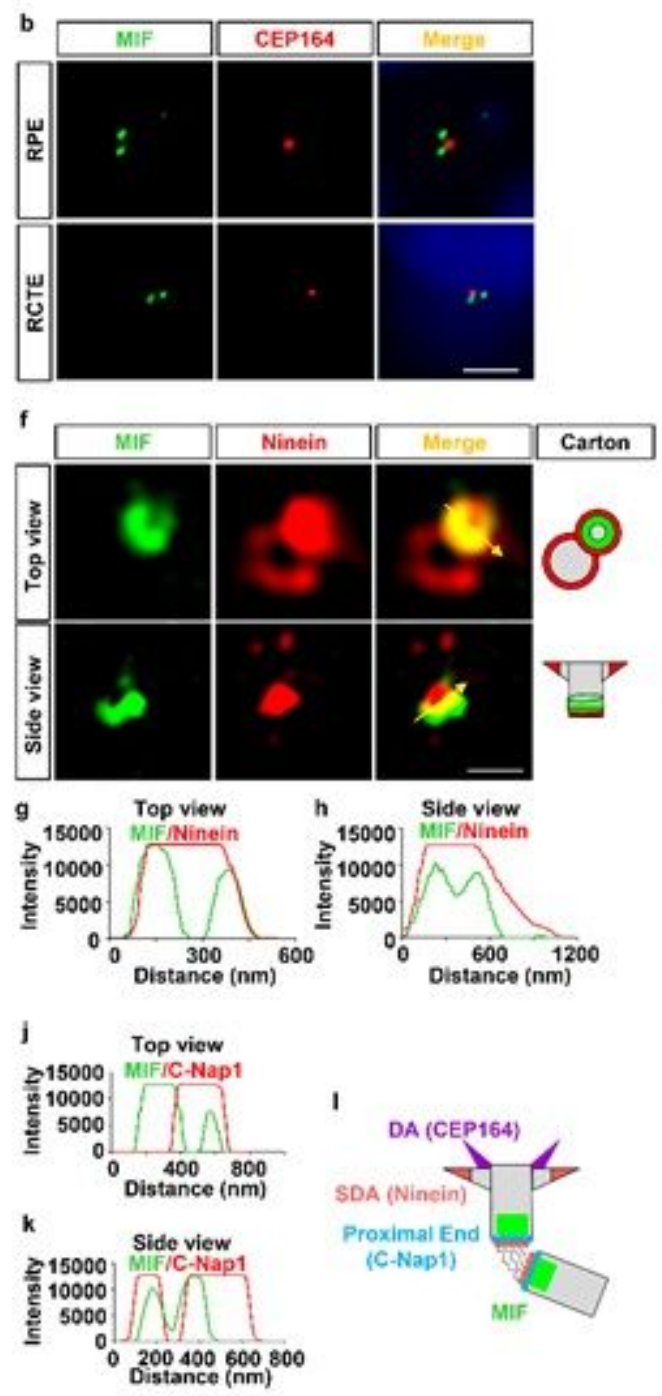

\section{Figure 2}

MIF is located and forms ring-like structures at the proximal ends of the centrioles. $a, b$ Representative images of RCTE cells stained with MIF (green) antibody and co-stained with Ninein (a) or CEP164 (b) under 2D microscope. Scale bars, $5 \mu \mathrm{m}$. c Representative images of RCTE cells stained with MIF (green) antibody and co-stained with CEP164 (red), which were visualized under 3D-SIM microscopy. Scale bars, $500 \mathrm{~nm}$. d, e The intensity plots of the rings in 3D images (c) from top (d)and side (e) views. $f$ Representative images of RCTE cells stained with MIF (green) antibody and co-stained with Ninein (red), which were visualized under 3D-SIM microscopy. Scale bars, $500 \mathrm{~nm} . \mathrm{g}$, h The intensity plots of the rings in 3D images ( $\mathrm{f}$ ) from top $(\mathrm{g})$ and side (h) views. i Representative images of RCTE cells stained with MIF 
(green) antibody and co-stained with C-Nap1 (red), which were visualized under 3D-SIM microscopy. Scale bars, $500 \mathrm{~nm}$. j, k The intensity plots of the rings in 3D images (i) from top (j) and side (k) views. I Schematic of the localization of MIF, CEP164, Ninein, and C-Nap1 at centrosomes. DA, distal appendage; SDA, subdistal appendage.
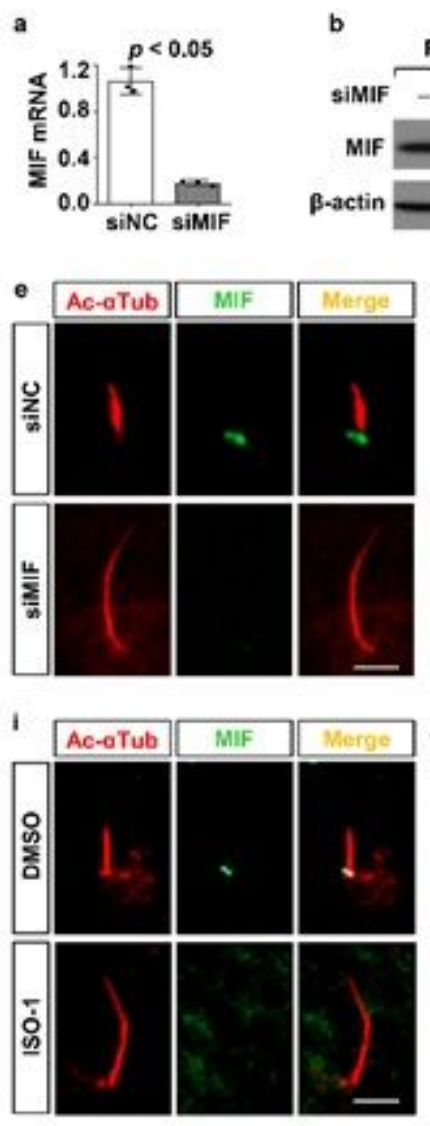
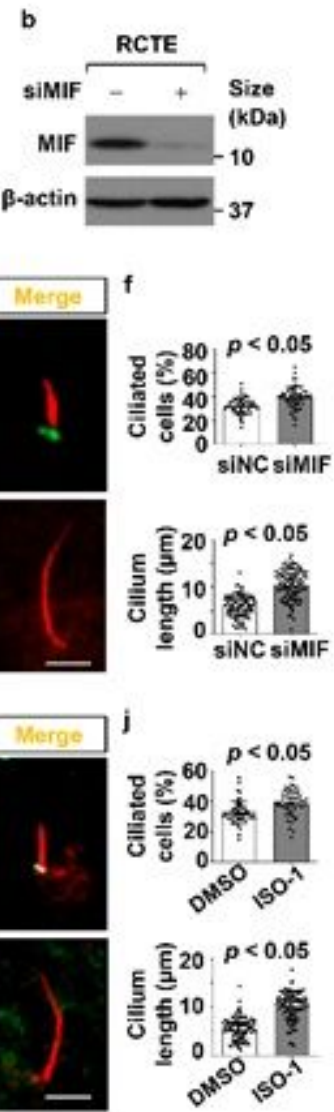
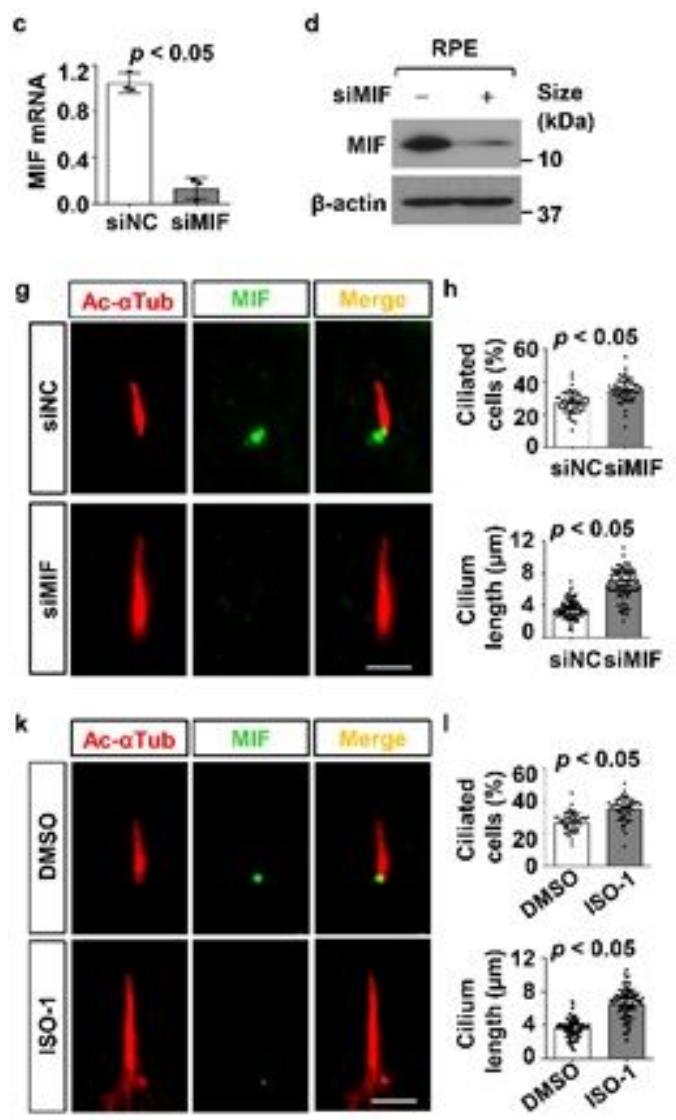

\section{Figure 3}

Knockdown and inhibition of MIF facilitated cilia assembly and elongation in RCTE and RPE cells. a-d The levels of MIF in RCTE cells $(a, b)$ and RPE cells $(c, d)$ transfection with MIF and control siRNA were examined with qRT-PCR $(a, c)$ and Western blot $(b, d)$ analysis. e Representative images of RCTE cells transfected with MIF and control siRNAs for $48 \mathrm{~h}$ and serum starved for $24 \mathrm{~h}$, and then stained with MIF (green) and acetyl- a-tubulin (red) antibodies. Scale bars, 5 mm. f Knockdown of MIF in RCTE cells (e) increased the percentage of ciliated cells (top) and cilia length (bottom). g Representative images of RPE cells transfected with MIF and control siRNAs for $48 \mathrm{~h}$ and serum starved for $24 \mathrm{~h}$, and then stained with MIF (green) and acetyl-a-tubulin (red) antibodies. Scale bars, $5 \mu \mathrm{m}$. h Knockdown of MIF in RPE cells (g) the percentage of ciliated cells (top) and cilia length (bottom). i Representative images of RCTE cells treated with DMSO or ISO-1 (100 $\mu \mathrm{M})$ plus serum free for $24 \mathrm{~h}$, and then stained with MIF (green) and acetyl-a-tubulin (red) antibodies. Scale bars, $5 \mu \mathrm{m}$. j Inhibition of MIF in RCTE cells (i) increased the percentage of ciliated cells (top) and cilia length (bottom). k Representative images of RPE cells treated with DMSO or ISO-1 (100 $\mu \mathrm{M})$ plus serum free for 24h, and then stained with MIF (green) and acetyl-atubulin (red) antibodies Scale bars, $5 \mu \mathrm{m}$. I Inhibition of MIF in RPE cells (k) increased the percentage of 
ciliated cells (top) and cilia length (bottom). $n=3$ biologically independent experiments. The ciliated cell percentages of about 50 views was analyzed in each group; The cilia lengths of about 100 cells was analyzed in each group $(f, h, j, I)$. All data are represented as the mean value \pm s.d. Significant differences were identified by student's t-test.

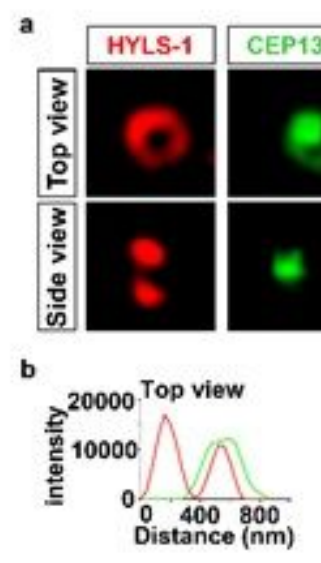

g

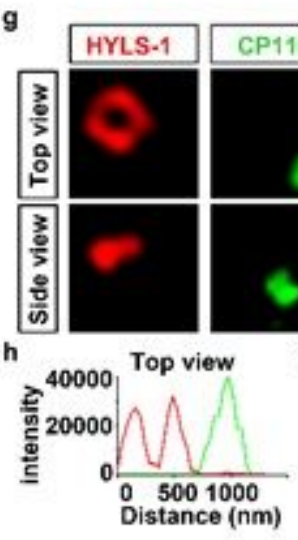

j
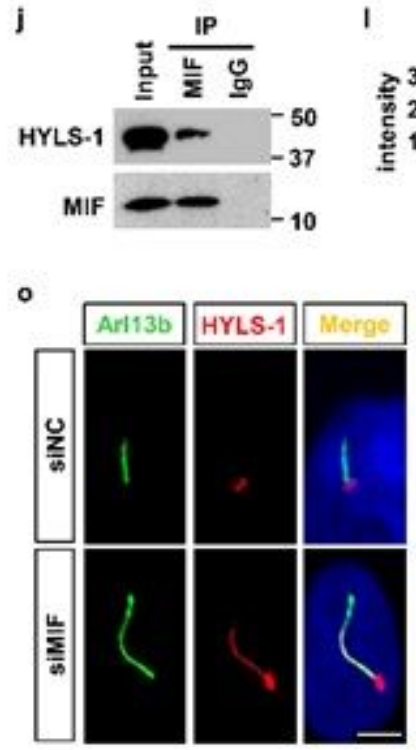
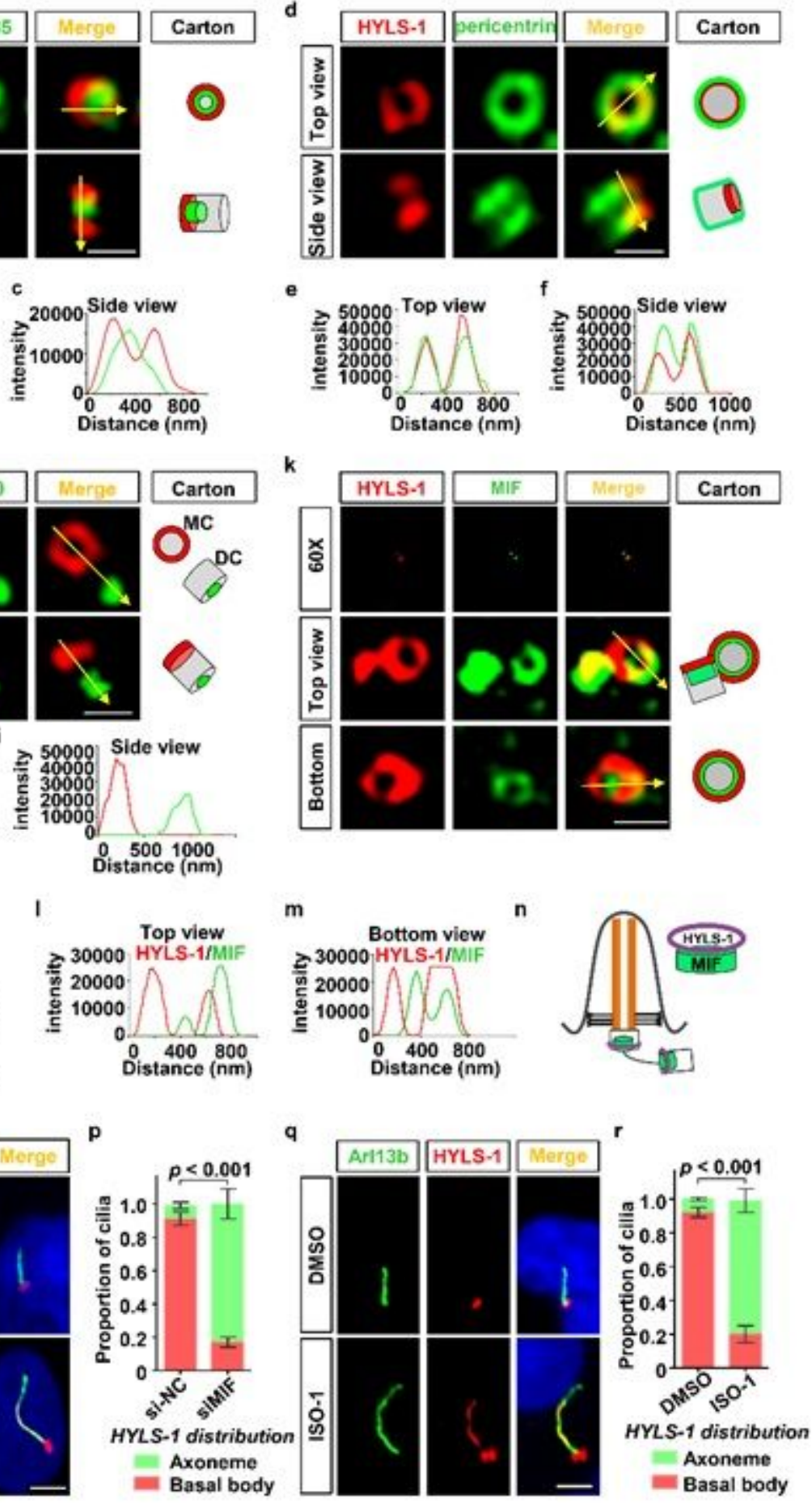

Figure 4

IF interacts with HYLS-1 and prevents HYLS-1 translocation from basal body into axoneme. a Representative image of RCTE cells stained with HYLS-1 (red) antibody and co-stained with CEP135 
(green), which were visualized under 3D-SIM microscopy. Scale bars, $500 \mathrm{~nm}$. b, c The intensity plots of the rings in 3D images (a) from top (b) and side (c) views. d Representative images of RCTE cells stained with HYLS-1 (red) antibody and co-stained with pericentrin (green), which were visualized under 3D-SIM microscopy. Scale bars, $500 \mathrm{~nm}$. e, f The intensity plots of the rings in 3D images (d) from top (e) and side (f) views. g Representative images of RCTE cells stained with HYLS-1 (red) antibody and co-stained with CP110 (green), which were visualized under 3D-SIM microscopy. Scale bars, $500 \mathrm{~nm}$. h, i The intensity plots of the rings in 3D images (g) from top $(\mathrm{h})$ and side (i) views. j Lysates from RCTE cells were subjected to immunoprecipitation (IP) with anti-MIF antibody, and immunoblotting with the indicated antibodies. k Representative images of RCTE cells stained with HYLS-1 (red) antibody and costained with MIF (green), which were visualized under 3D-SIM microscopy. Scale bars, $500 \mathrm{~nm}$. I, m The intensity plots of the rings in 3D images $(\mathrm{k})$ from top $(\mathrm{I})$ and bottom $(\mathrm{m})$ views. $n$ Diagram to illustrate the relative localization of MIF and HYLS-1 at the proximal ends of centrioles. o Representative images of RCTE cells transfected with MIF and control siRNAs for $48 \mathrm{~h}$ and serum starved for $24 \mathrm{~h}$, and stained with ARL13B (green) and HYLS-1 (red) antibodies and co-stained with DAPI (blue). Scale bars, $5 \mu \mathrm{m} . \mathrm{p}$ Stacked bar graph represented the distribution of HYLS-1 along axoneme in RCTE cells transfected with MIF and control siRNAs. q Representative images of RCTE cells treated with DMSO or ISO-1 and stained with ARL13B (green) and HYLS-1 (red) antibodies and co-stained with DAPI (blue). Scale bars, $5 \mu \mathrm{m}$. r Stacked bar graph represented the distribution of HYLS-1 along axoneme in RCTE cells treated with ISO-1 and DMSO. $n=3$ biologically independent experiments. About 50 cells was analyzed in each group $(p, r)$. Data values are presented as mean \pm SEM. Statistics were performed using one-sided one-way ANOVA followed by post hoc LSD test (p, r). 
a

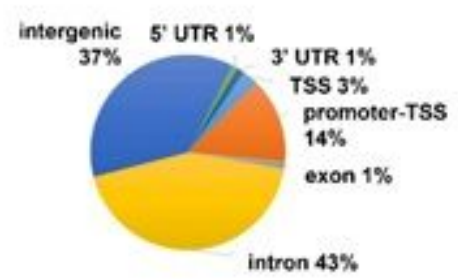

c

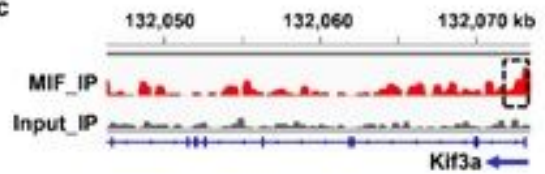

e

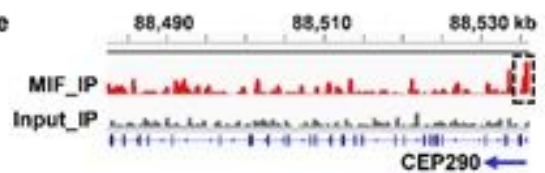

g

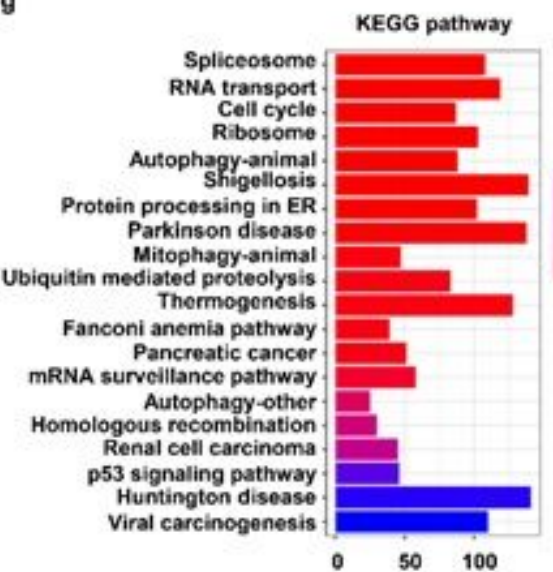

b

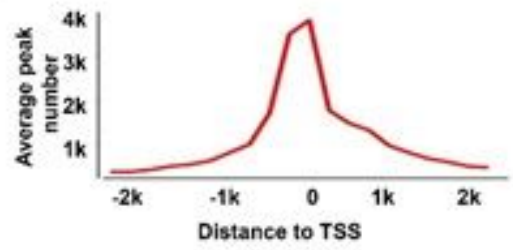

d

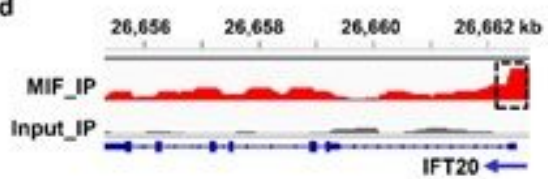

I

h

GO cellular component

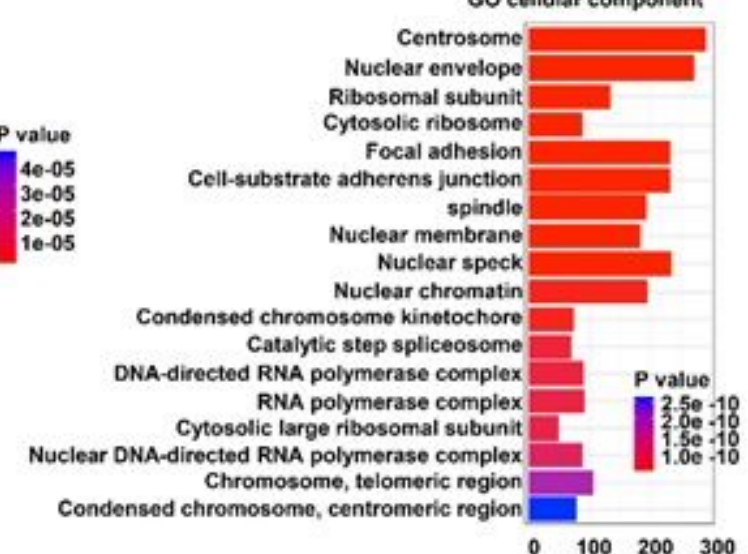

\section{Figure 5}

Identification of MIF target genes with ChIP-seq analysis. a The distribution of MIF ChIP-peaks was across different genomic regions. The pie chart shows that MIF binds to the promoters-TSS (transcription start site) region in about $14 \%$ of its target genes. b The distribution of promoter peaks identified with MIF ChIP-seq was markedly concentrated near the TSS. c-f Peaks of MIF occupancy on the promoters of KIF3a (c), IFT20 (d), CEP290 (e) and BBS4 (f) were identified by manual inspection and were showed as the input and MIF ChIP. g, h KEGG pathway and GO categories analysis of the MIF target genes. 


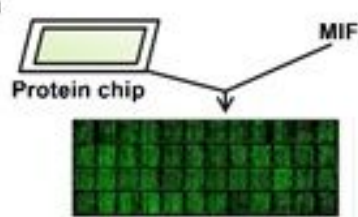

MIF-interacting proteins

b

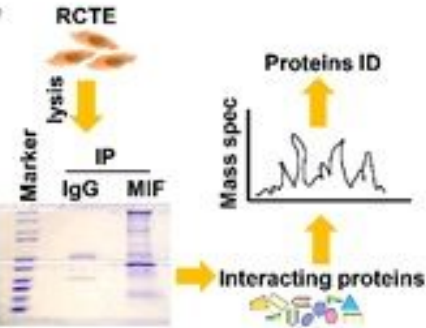
Human 80-LCGLLAERLRISPDRVYN-98 Mouse 80-LCGLLSDRLHISPDRVYIN-98 Rat 80-LCGLLSDRLHISPDRVYIN-98 14-3-3 binding motif - R-X-X-X-S

h

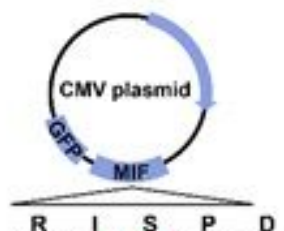

WT: 5 '- CGC ATC $\overline{\text { AGC }}$ CCG GAC -3 ' Mut: $5^{\prime}$-. CGC ATC GAC CCG GAC-3' R I $\frac{\text { D }}{P}$ D

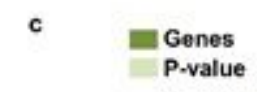

Protein ubiquitination Protein phosphorylation Apoptotic process $\quad 7 \quad 7 \quad 0.0459$ Cell proliferation $\quad 8 \quad 0.001 /$ \begin{tabular}{lll} 
MAPK cascade & 8 & 0.001 \\
\hline
\end{tabular}

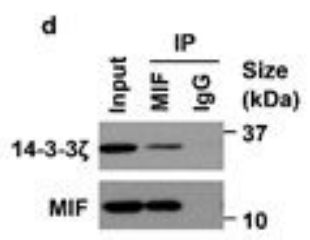

$\mathbf{f}$

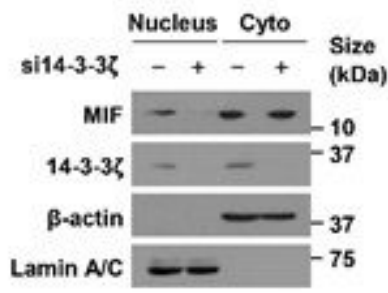

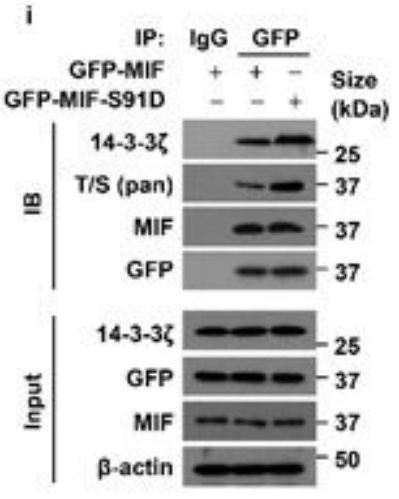

j

m

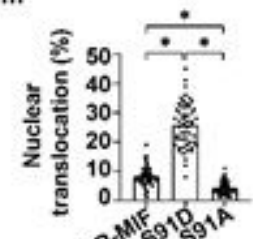

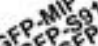

k

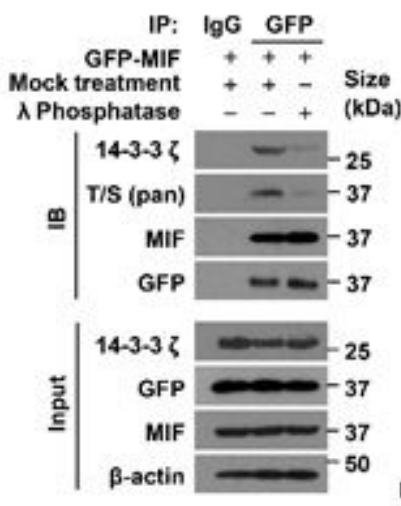

I
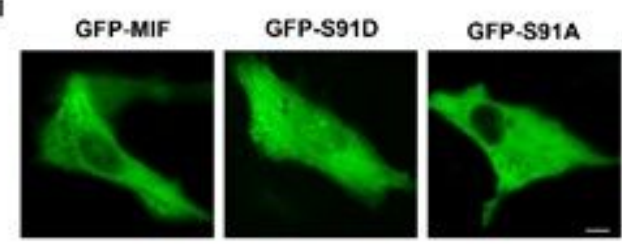

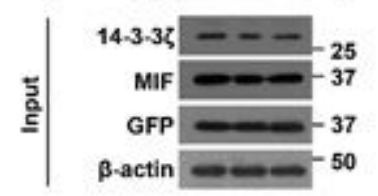

IP: IgG GFP

GFP-MIF + + - Size

GFP-MIF-S91A $-\ldots+(k D a)$

$\underline{\underline{9}}$

$14-3-3 \zeta$
T/S (pan)

MIF -37

GFP $=-37$
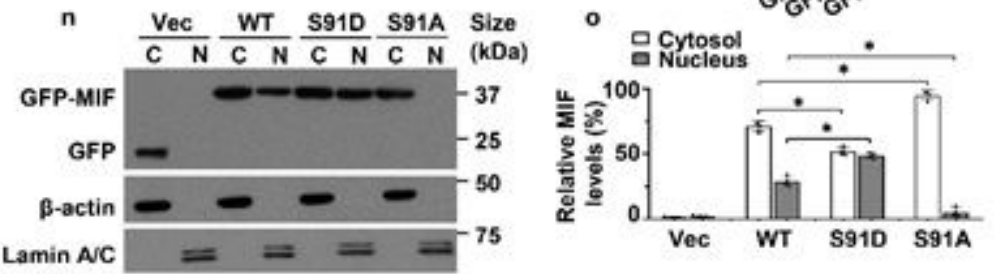

\section{Figure 6}

The translocation of MIF to nucleus is dependent on its phosphorylation and interaction with 14-3-3Z. a Identification of MIF-associated proteins by protein chip. b Identification of MIF-associated proteins by immunoprecipitation with MIF antibody and followed by mass spectrometry (MS) in RCTE cells. c MIF associated proteins identified by MS were clustered with a range of gene ontology (GO) biological processes by functional annotation of MIF binding sites using the Genomic Regions Enrichment of Annotations Tool (GREAT). d Immunoprecipitation assay was performed to detect the interaction between MIF and 14-3-3ろ. e The nuclear and cytoplasmic fractions were isolated from RCTE cells and then those 
fractions were subjected to immunoprecipitation with MIF antibody and IgG, and followed by immunoblotting (IB) with indicated antibodies. f Western blot analysis of MIF and 14-3-3 3 in nuclear and cytoplasmic fractions isolated RCTE cells transfected with 14-3-3 3 and control siRNAs. g Alignment of MIF sequences from different species (Human, Mouse, Rat). Highlighted letters indicate the conserved canonical 14-3-3 3 binding motif. $h$ The schematic diagram shows the mutation of S91D in GFP tagged MIF plasmid. i Immunoprecipitation assay to detect the phosphorylation of MIF and the interaction between GFP tagged MIF and 14-3-3Z in RCTE cells transfected with GFP tagged wild type MIF and GFP tagged mutant MIF-S91D plasmids. j Immunoprecipitation assay to detect the phosphorylation of MIF and the interaction between GFP tagged MIF and 14-3-3ろ in RCTE cells transfected with GFP tagged wild type MIF and GFP tagged mutant MIF-S91A plasmids. k Immunoprecipitation assay to detect the phosphorylation of MIF and the interaction between GFP tagged MIF and 14-3-3 $\zeta$ in RCTE cells treated with $\lambda$-phosphatase and vehicle (control). I Representative images of HEK293 cells transfected with GFPtagged wild type MIF and GFP-tagged mutant MIF (S91D or S91A). Scale bars, $5 \mu \mathrm{m}$. m Quantification of the percentage of cells with nuclear translocation of MIF in HEK293T cells transfected with GFP-tagged wild type MIF and GFP-tagged mutant MIF (S91D or S91A). About 50 cells was analyzed in each group $(\mathrm{m})$. $\mathrm{n}$ Western blot analysis of GFP tagged MIF in nuclear (N) and cytoplasmic (C) fractions isolated HEK293T cells transfected with GFP-tagged wild type MIF and GFP-tagged mutant MIF (S91D or S91A). o Statistical analysis of relative levels of MIF in cytosol and nuclear fractions isolated HEK293T cells transfected with GFP-tagged wild type MIF and GFP-tagged mutant MIF (S91D or S91A) from three independent experiments. All data are represented as the mean value \pm s.d. Significant differences were identified by student's t-test. ${ }^{*} \mathrm{p}<0.05$. 

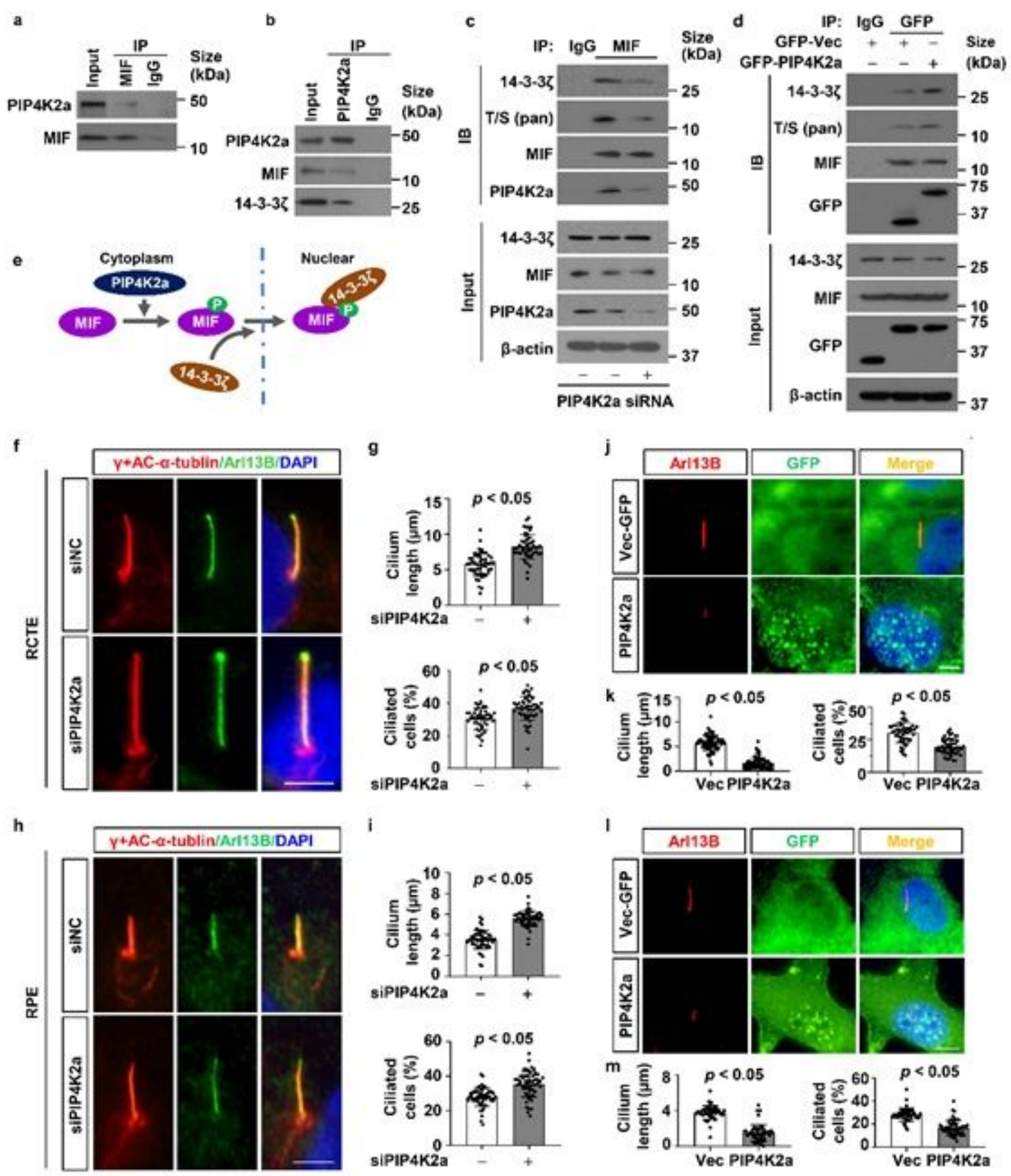

\section{Figure 7}

Phosphorylation of MIF at Ser91 is regulated byPIP4K2a. a, b Lysates from RCTE cells were subjected to immunoprecipitation (IP) with anti-MIF (a) and anti-PIP4K2a (b) antibodies, and immunoblotting with the indicated antibodies. $\mathrm{c} \mathrm{Immunoprecipitation} \mathrm{assay} \mathrm{to} \mathrm{detect} \mathrm{the} \mathrm{phosphorylation} \mathrm{of} \mathrm{MIF} \mathrm{and} \mathrm{the}$ interaction between MIF and 14-3-3ろ in RCTE cells transfected PIP4K2a and control siRNAs. $d$ Immunoprecipitation assay to detect the phosphorylation of MIF and the interaction between GFP-MIF and 14-3-3 $\zeta$ in RCTE cells transfected with GFP-Vector and GFP tagged PIP4K2a plasmids. e Schematic diagram indicates that PIP4K2a phosphorylates MIF at S91 and phospho-MIF interacts with 14-3-3Z to promote the nuclear translocation of MIF. f Representative images of RCTE cells transfected with PIP4K2a and control siRNAs for 48h and then serum starved for 24h, and stained with ARL13B (green), $\mathrm{Y}^{-}$ 
tubulin and acetyl-a-tubulin (red) antibodies and co-stained with DAPI (blue). Scale bars, $5 \mu \mathrm{m} . \mathrm{g}$ Knockdown of PIP4K2a in RCTE cells (f) increased cilia length (top) and the percentage of ciliated cells (bottom), which were quantified and plotted from three independent experiments. $h$ Representative images of RPE cells transfected with PIP4K2a and control siRNAs for $48 \mathrm{~h}$ and serum starved for $24 \mathrm{~h}$, and then stained with ARL13B (green), $y$-tubulin and acetyl-a-tubulin (red) antibodies and co-stained with DAPI (blue). Scale bars, $5 \mu \mathrm{m}$. i Knockdown of PIP4K2a in RPE cells (h) increased cilia length (top) and the percentage of ciliated cells (bottom), which were quantified and plotted from three independent experiments. $j$ Representative images of RCTE cells transfected with GFP-vector and GFP tagged PIP4K2a plasmids for $48 \mathrm{~h}$ and then serum starved for 24h, and stained with ARL13B (red) and GFP (green) antibodies and co-stained with DAPI (blue). Scale bars, $5 \mu \mathrm{m}$. k Overexpression of GFP tagged PIP4K2a in RCTE cells decreased cilium length (left) and percentage of ciliated cells (right) compared to those in cells transfected with GFP-vector, which were quantified and plotted from three independent experiments. I Representative images of RPE cells transfected with GFP-vector and GFP tagged PIP4K2a plasmids for $48 \mathrm{~h}$ and then serum starved for 24h, and stained with ARL13B (red) and GFP (green) antibodies and costained with DAPI (blue). Scale bars, $5 \mu \mathrm{m}$. m Overexpression of GFP tagged PIP4K2a in RPE cells decreased cilium length (left) and percentage of ciliated cells (right) compared to those in cells transfected with GFP-vector, which were quantified and plotted from three independent experiments. The ciliated cell percentages of about 50 views was analyzed in each group; The cilia lengths of about 100 cells was analyzed in each group $(\mathrm{g}, \mathrm{k}, \mathrm{i}, \mathrm{m})$. All data are represented as the mean value $\pm \mathrm{s}$.d. Significant differences were identified by student's t-test. 


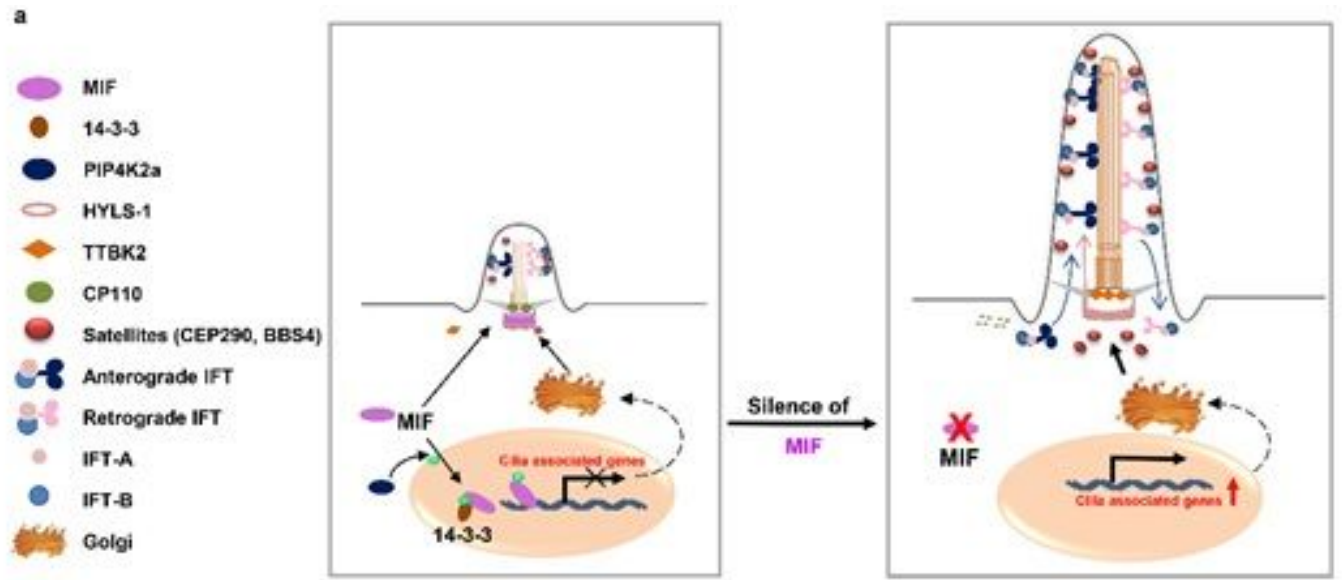

b

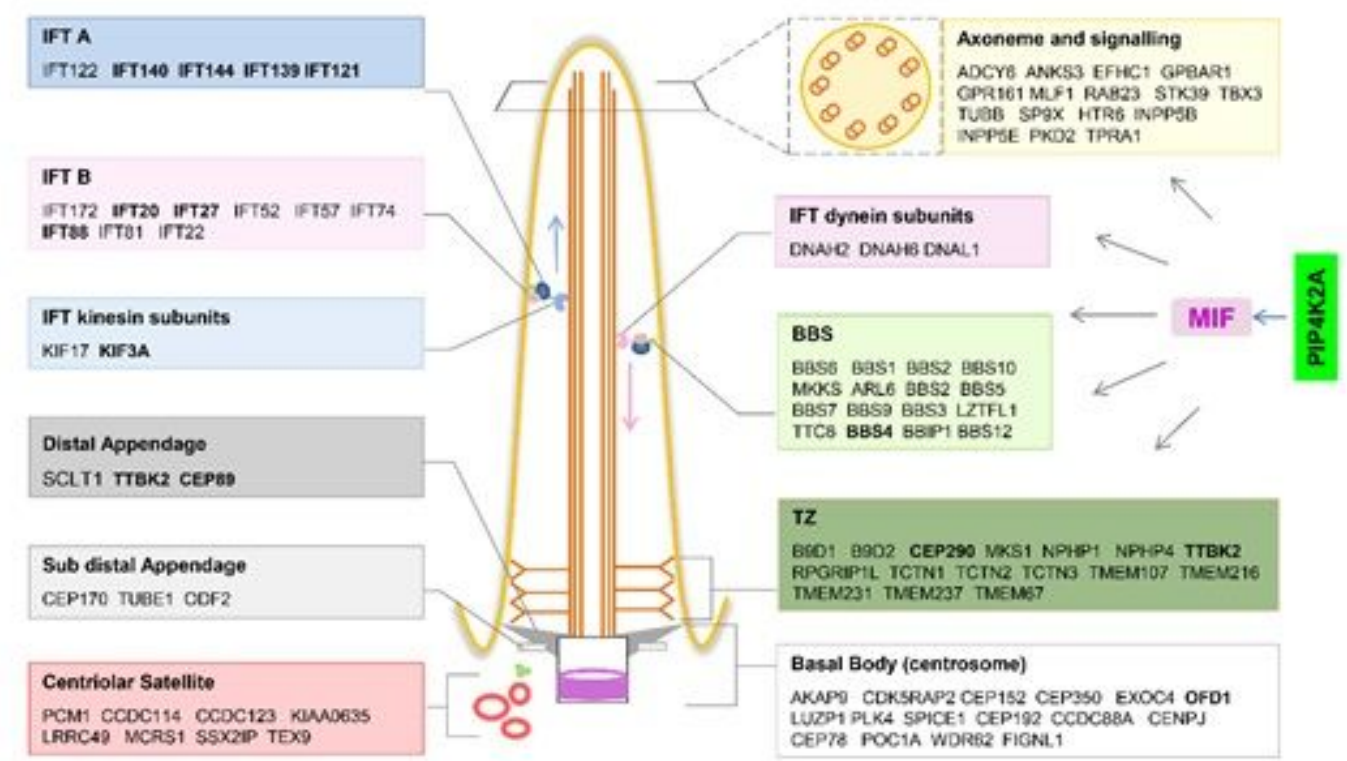

Figure 8

Working model. a MIF is localized at basal body, which forms a ring like structure and partially colocalized with HYLS-1 at proximal ends of centrioles in mammalian cells. MIF regulates cilia assembly and elongation via affecting, 1) the removal of CP110 from mother centriole and the recruitment of TTBK2 to basal body, 2) the accumulation of CEP290 and BBS4 at centriolar satellites, 3) the trafficking of KIF3a and IFT particles, including IFT20, IFT88 and IFT140 (left). Depletion of MIF promotes cilia initiation through TTBK2-CP110-CEP290-BBS4 axis and cilia elongation through an increase of the trafficking and entry of HYLS-1, KIF3a, IFT20 and IFT88 into axoneme and a decrease of IFT140 at cilia tip (right). Importantly, MIF can be phosphorylated by PIP4K2a and phospho-MIF interacts with 14-3-3Z, both of which facilitate the translocation of MIF into nucleus (left). The nuclear MIF binds to the promoters of its target genes, such as genes associated with cilia biogenesis, to negatively regulate their transcription. Thus, depletion of MIF may also promote cilia biogenesis through releasing its inhibition on the repression of ciliary genes which are identified by ChIP-seq analysis and are grouped based on their localization and roles in ciliogenesis in panel $b$. 


\section{Supplementary Files}

This is a list of supplementary files associated with this preprint. Click to download.

- Supplementaryinformations.pdf

- Reportingsummary.pdf

- SupplementaryData1ciliarelatedgenespromoterTSS.xIsx

- SupplementaryData2PromoterTTSotherthanciliogenesis.xlsx

- SupplementaryData3ProteinChip.xlsx

- SupplementaryData4MS.xIsx 